H. Umemura

Nagoya Math. J.

Vol. 52 (1973) $97-128$

\title{
SOME RESULTS IN THE THEORY OF VECTOR BUNDLES
}

\author{
HIROSHI UMEMURA
}

We have several definitions of the positivity of a vector bundle, differentiable definitions, an algebro-geometric definition, a topological definition etc. In $\S 1$ we review the definitions and the relations between them. For a line bundle all the definitions are equivalent and every one agrees that they are reasonable. For a vector bundle, however, the definitions are not necessarily equivalent. One of the main results of this paper is the equivalence of the definitions over a complete nonsingular curve. The proof is given in $\S 2$. We proved this over an elliptic curve in Umemura [18]. In this case the proof was based on Atiyah's classification. To prove the equivalence over a curve of genus $\geq 2$, the fundamental lemma is; A stable bundle of positive degree is positive in the sense of Nakano. The tool used to prove this lemma is the theory of stable bundles due to Narasimhan and Seshadri [11] - they establish a correspondence between stable bundles and certain types of irreducible unitary representations of a Fuchsian group.

We also discuss the $H$-stability of Takemoto from two points of view. In $\S 3$, we prove that over an abelian surface, a ruled surface or a hyperelliptic surface, $H$-stable bundle of rank 2 with $c_{1}^{2}-4 c_{2} \geq 0, c_{1}>0$, $c_{2}>0$ is positive in the sense of Nakano. We ask in general: Is a stable bundle of rank 2 over a surface with positive Chern class ample? This is the analogue of the lemma that we mentioned above. But this is false unless $c_{1}^{2}-4 c_{2} \geq 0$, even over an abelian surface. Hence $H$-stability is not very comfortable in this case.

In $\S 4$, we deal with vanishing theorems. The first theorem is well known as the index theorem. In fact an algebraic proof is known. The second theorem (4.2) is a generalization of the Kodaira vanishing theorem and Mumford's result [8]. We also remark that a vanishing theorem

Received June 7, 1973. 
of Griffiths is proved easily by the standard argument using cohomological dimension and the spectral sequence of de Rham cohomology.

In $\S 5$ we study $H(E)$ and $\mathscr{G}(E)$ for a vector bundle $E$. In general $H(E)$ and $\mathscr{G}(E)$ are too small. So we have to find a good family of vector bundles $E$ such that $H(E)$ and $\mathscr{G}(E)$ reflect properties of $E$. Over an elliptic curve, if $E$ is stable, $H(E)$ and $\mathscr{G}(E)$ are nice and give the Heisenberg group. So it is quite natural to ask if $H(E)$ and $\mathscr{G}(E)$ give sufficient information concerning $E$ for an $H$-stable bundle $E$ over an abelian surface. Unfortunately the answer is no unless $c_{1}^{2}-4 c_{2}=0$. Here again the $H$-stability with $c_{1}^{2}-4 c_{4}<0$ is unpleasant.

\section{§1. Preliminaries}

(1.1) Let $V$ be a non-singular projective algebraic variety of dimension $n$ defined over the complex number field $C$. Let $E$ be a holomorphic vector bundle of rank $r$ defined over $V$. Let $\left\{U_{\alpha}\right\}$ be an open covering of $V$ such that $E$ is trivial on each $U_{\alpha}$. Let $g_{\beta \alpha}$ be the transition matrix of $E$ i.e. two elements $x \times \xi_{\alpha}$ and $x \times \xi_{\beta}$ with $x \in U_{\alpha} \cap U_{\beta}, \xi_{\alpha}, \xi_{\beta} \in C^{r}$ are identified if and only if $g_{\beta \alpha}(x) \xi_{\alpha}=\xi_{\beta}$.

A hermitian metric on $E$ is, by definition, a set of $C^{\infty}$-maps $h_{\alpha}$ from $U_{\alpha}$ to the space of positive definite hermitian matrices of degree $r$ such that ${ }^{t} \bar{g}_{\beta \alpha}(z) h_{\alpha}(z) g_{\beta \alpha}(z)=h_{\beta}(z)$ for any $z \in U_{\alpha} \cap U_{\beta}$.

A $\left(C^{\infty}-\right)$ connection on $E$ is a set of 1 -forms $\theta_{\alpha}$ on $U_{\alpha}$ such that $\theta_{\alpha}$ $=g_{\alpha \beta}\left(\theta_{\beta}-\omega_{\alpha \beta}\right) g_{\alpha \beta}^{-1}$ on $U_{\alpha} \cap U_{\beta}$ where $\omega_{\alpha \beta}=g_{\alpha \beta}^{-1} d g_{\alpha \beta}$. It is easy to see that a connection defines a $C$-linear map $D: E \rightarrow \Omega^{1}(E)$ by putting

$$
D\left(\varphi_{\alpha}\right)=d \varphi_{\alpha}+\theta_{\alpha} \wedge \varphi_{\alpha}
$$

for a local section $\varphi_{\alpha}$ on each $U_{\alpha}$. Similarly we can define two operators:

$$
\begin{aligned}
D^{\prime}: E & \rightarrow \Omega^{1}(E) \\
\varphi_{\alpha} & \mapsto d^{\prime} \varphi_{\alpha}+\theta_{\alpha} \wedge \varphi_{\alpha}, \\
D^{\prime \prime}: E & \rightarrow \Omega^{1}(E) \\
\varphi_{\alpha} & \mapsto d^{\prime \prime} \varphi_{\alpha}
\end{aligned}
$$

so that $D=D^{\prime}+D^{\prime \prime}$.

$D$ induces an operator from $\Omega^{p}(E)$ to $\Omega^{p+1}(E)$ by the following formula

$$
D(\lambda, \varphi)=d \lambda \cdot \varphi+(-1)^{p} \lambda \wedge D \varphi,
$$

where $\lambda$ is a local section of $\Omega^{p}$ and $\varphi$ is a local section of $E$. We 
denote this operator also by $D . \quad D^{2}$ is called the curvature form of the connection $\theta=\left\{\theta_{\alpha}\right\}$.

Let $h=\left\{h_{\alpha}\right\}$ be a hermitian metric on $E$. Then by an easy calculation, we see that $\left\{h_{\alpha}^{-1} d^{\prime} h_{\alpha}\right\}$ defines a connection on $E$. Let $\left\{\Theta_{\alpha}\right\}$ be the curvature form of the connection $h^{-1} d^{\prime} h$. More explicitely $\Theta_{\alpha}=d \theta_{\alpha}+$ $\theta_{\alpha} \wedge \theta_{\alpha}=-h_{\alpha}^{-1} d^{\prime} d^{\prime \prime} h_{\alpha}-h_{\alpha}^{-1} d^{\prime \prime} h_{\alpha} \wedge h_{\alpha}^{-1} d^{\prime} h_{\alpha}$. We put

$$
\Theta_{\xi}(\eta)=\sum_{\substack{1 \leq \rho, \tau, \sigma \leq r \\ 1 \leq i, j \leq n}} h_{\tau}^{\rho} \Theta_{\sigma i j}^{\tau} \xi^{\rho} \bar{\xi}^{\sigma} \eta^{i} \bar{\eta}^{j}
$$

where $\quad \xi={ }^{t}\left(\xi_{1}, \cdots, \xi_{r}\right) \in \boldsymbol{C}^{r}, \eta={ }^{t}\left(\eta_{1}, \cdots, \eta_{n}\right) \in \boldsymbol{C}^{n}, \Theta=\left(\sum_{1 \leq i, j \leq n} \Theta_{\sigma i j}^{\rho} d z_{i} \wedge\right.$ $\left.d \bar{z}_{j}\right)_{1 \leq \rho, o \leq r}$ and $z_{1}, \cdots, z_{n}$ is a local coordinate system on $V$ (we drop the index $\alpha$ when no confusion is possible).

We say that a vector bundle $E$ is positive (resp. negative) and denote it by $E>0$ (resp. $E<0$ ) if there exists a hermitian metric $h$ on $E$ such that $\Theta_{\xi}(\quad)$ is positive (resp. negative) definite any point $P$ of $V$ for any $\xi \neq 0$.

A vector bundle $E$ is non-negative (resp. non-positive) and we denote it by $E \geq 0$ (resp. $E \leq 0$ ) if there exists a hermitian metric $h$ such that $\Theta_{\xi}(\quad)$ is non-negative (non-positive) at any point $P$ of $V$ for any $\xi \neq 0$.

Remark (1.2) Let $P$ be a point of $V$. By choosing a frame, we may assume $d^{\prime} h(P)=0$ and $h(P)=I$. Then the curvature form at $P$ is equal to $-d^{\prime} d^{\prime \prime} h$.

Remark (1.3) Let $\left\{h_{\alpha}\right\}$ be a metric on $E$. If we put $\varphi_{\alpha}=\sum h_{\alpha t}^{s} \xi^{s} \bar{\xi}^{t}$ with $\xi={ }^{t}\left(\xi_{1}, \cdots, \xi_{r}\right) \in C^{r}$ on each $U_{\alpha} \times C^{r}$, then $\varphi_{\alpha}$ is a well defined function. Let $P$ be a point of $U_{\alpha}$. We normalize $h$ at $P$ as in Remark

(1.2). Then the Levi form of $\varphi$ at $P \times \xi$ is equal to

$$
\left(\begin{array}{cccc}
\sum_{1 \leq s, t \leq r} \frac{\partial^{2} h_{t}^{s}}{\partial z_{1} \partial \bar{z}_{1}} \xi^{s} \bar{\xi}^{t} & \cdots & \sum_{1 \leq s, t \leq r} \frac{\partial^{2} h_{t}^{s}}{\partial z_{1} \partial \bar{z}_{n}} \xi^{s \bar{\xi}^{t}} & \\
\vdots & & \vdots & 0 \\
\sum_{1 \leq s, t \leq r} \frac{\partial^{2} h_{t}^{s}}{\partial z_{n} \partial \bar{z}_{1}} \xi^{s} \bar{\xi}^{t} & \cdots & \sum_{1 \leq s, t \leq r} \frac{\partial^{2} h_{t}^{s}}{\partial z_{n} \partial \bar{z}_{n}} \xi^{s} \bar{\xi}^{t} & \\
0 & & & h
\end{array}\right)
$$

Hence $\#\left\{\right.$ negative eigenvalues of $\Theta_{\xi}(\quad)$ at $\left.P\right\}=n-\#\{$ negative eigenvalues of the Levi form of $\varphi$ at $P$ \} 
DEFINITION (1.4) A vector bundle $E$ is positive (resp. non-negative) in the sense of Nakano if there exists a hermitian metric $h$ on $E$ such that

$$
\sum_{\substack{1 \leq \rho, \tau, \sigma \leq r \\ 1 \leq i, j \leq n}} h_{\tau}^{\rho} \Theta_{\sigma i j}^{\tau} \xi^{(\rho, i)} \overline{\xi^{(\sigma, j)}}
$$

is positive (resp. non-negative) at any point for any non-zero vector $\left(\cdots \xi^{(\rho, i)} \cdots\right) \in C^{n r}$.

LEMMA (1.5) If a vector bundle is positive in the sense of Nakano, then it is positive. The converse holds if either $r=1$ or $n=1$.

Proof. Trivial from the definition.

Remark (1.6). A positive vector bundle is not positive in the sense of Nakano in general.

LEMMA (1.7) Let $E$ be a vector bundle on $V$. Let $h$ be a hermitian metric on $E$. Then $h$ induces a natural hermitian metric $\breve{h}$ on $\check{E}$. We have $\Theta=-^{t \check{\Theta}}$ where $\Theta$ (resp. $\left.\check{\Theta}\right)$ is the curvature form of $h$ (resp. $\check{h}$ ).

Proof. Let $\left\{h_{\alpha}\right\}$ define a metric on $E$. Then $\left\{{ }^{t} h_{\alpha}^{-1}\right\}=\left\{\check{h}_{\alpha}\right\}$ is a metric on $\check{E}$. In fact.

$$
\begin{gathered}
{ }^{t} \bar{g}_{\beta \alpha} h_{\alpha} g_{\beta \alpha}=h_{\beta} \\
\bar{g}_{\alpha \beta}^{-1}{ }^{t} h_{\alpha}^{-1}{ }^{t} g_{\beta \alpha}^{-1}={ }^{t} h_{\beta}^{-1} \\
{ }^{t}\left({ }^{t} g_{\beta \alpha}^{-1}\right)^{t} h_{\alpha}^{-1}{ }^{t} g_{\beta \alpha}^{-1}={ }^{t} h_{\beta}^{-1}
\end{gathered}
$$

Let $\check{\theta}$ be the connection of $\left\{{ }^{t} h_{\alpha}^{-1}\right\}$. Then

$$
\begin{aligned}
\check{\theta}_{\alpha} & =\left({ }^{t} h^{-1}\right)^{-1} d^{t} h^{-1} \\
& =-d^{t} h \cdot{ }^{t} h^{-1} \\
& =-{ }^{t}\left(h^{-1} d^{\prime} h\right) \\
& =-{ }^{t} \theta .
\end{aligned}
$$

Hence $\check{\Theta}_{\rho}^{\sigma}=-\Theta_{\sigma}^{\rho}$.

ExAmPle (1.8) Projective space $P^{n}$. We put $U_{i}=\left\{\left(x_{0}, x_{1}, \cdots, x_{n}\right)\right.$ $\left.\in P^{n} \mid x_{i} \neq 0\right\}$ for $0 \leq i \leq n$. The transition function $g_{j i}$ of $O(-1)$ is $x_{i} / x_{j}$. If we put $h_{i}=\left|x_{0} / x_{i}\right|^{2}+\left|x_{1} / x_{i}\right|^{2}+\cdots+\left|x_{n} / x_{i}\right|^{2}$, then $h_{j}=\left.\left|x_{i}\right| x_{j}\right|^{2} h_{i}$ $=\left|g_{j i}\right|^{2} h_{i}$. Hence $\left\{h_{i}\right\}$ defines a metric on $O(-1)$. If we put $z_{1}=x_{0} / x_{i}$, $z_{2}=x_{1} / x_{i}, \cdots, z_{i}=x_{i-1} / x_{i}, z_{i+1}=x_{i+1} / x_{i}, \cdots, z_{n}=x_{n} / x_{i}$, the connection and the curvature form on $U_{i}$ are given by 


$$
\begin{gathered}
h^{-1} d^{\prime} h=\frac{\bar{z}_{1} d z_{1}+\bar{z}_{2} d z_{2}+\cdots+\bar{z}_{n} d z_{n}}{1+\left|z_{1}\right|^{2}+\left|z_{2}\right|^{2}+\cdots+\left|z_{n}\right|^{2}} \\
\Theta=\frac{-\left(1+\left|z_{1}\right|^{2}+\cdots+\left|z_{n}\right|^{2}\right)\left(d z_{1} \wedge d \bar{z}_{1}+\cdots+d z_{n} \wedge d \bar{z}_{n}\right)+}{1+\left|z_{1}\right|^{2}+\cdots+\left|z_{n}\right|^{2}} \\
+\left(\bar{z}_{1} d z_{1}+\cdots+\bar{z}_{n} d z_{n}\right)\left(z_{1} d \bar{z}_{1}+\cdots+z_{n} d \bar{z}_{n}\right) .
\end{gathered}
$$

Hence $O(-1)$ is negative and $O(1)$ is positive.

EXAmPle (1.9) An abelian variety. Let $A=C^{n} / \Gamma$ be an abelian variety where $\Gamma$ is a lattice in $C^{n}$. Since $C^{n}$ is Stein and simply connected, every line bundle is defined by an element of $H^{1}\left(\Gamma, H^{0}\left(\boldsymbol{C}^{n}, O_{\boldsymbol{C}^{n}}^{*}\right)\right)$ i.e. by a cocycle $u \mapsto e_{u}(z)$ for $\Gamma$ with coefficients in $H^{0}\left(C^{n}, O_{\boldsymbol{C}^{n}}^{*}\right)$ :

$$
e_{u+u^{\prime}}(z)=e_{u}\left(z+u^{\prime}\right) \cdot e_{u^{\prime}}(z) .
$$

Let $H$ be a hermitian form on $C^{n}$ such that $E=\operatorname{Im} H$ is integral on $\Gamma$. Let $\alpha: \Gamma \rightarrow\{z \in C|| z \mid=1\}$ be a map such that $\alpha\left(u_{1}+u_{2}\right)=e^{i \pi E\left(u_{1}, u_{2}\right)}$. $\alpha\left(u_{1}\right) \cdot \alpha\left(u_{2}\right), u_{i} \in \Gamma$. Then $u \mapsto e_{u}(z)=\alpha(u) e^{\pi H(z, u)+(1 / 2) \pi H(u, u)}$ is a cocycle for $\Gamma$ with coefficients in $H^{0}\left(C^{n}, O_{C^{n}}^{*}\right)$. Hence $\left\{e_{u}(z)\right\}$ determines a line bundle $L(H, \alpha)$. The theorem of Appell-Humbert says that any line bundle on $A$ is uniquely determined by a pair, $(H, \alpha)$ satisfying the condition above.

Let $L=L(H, \alpha)$ be a line bundle on $A$. If we put $\varphi(z)=e^{-\pi H(z, z)}$, $z \in \boldsymbol{C}^{n}$, then $\varphi(z)$ is a metric on the trivial line bundle $\boldsymbol{C}^{n} \times \boldsymbol{C}$ on $\boldsymbol{C}^{n}$. Let $e_{u}(z)$ be the cocycle defined by $(H, \alpha)$, then we have

$$
\varphi(z+u)\left|e^{\pi H(z, u)+(\pi / 2) H(u, u)}\right|^{2}=\varphi(z) .
$$

In fact,

$$
\begin{aligned}
\varphi(z+ & u)\left|e^{\pi H(z, u)+(\pi / 2) H(u, u)}\right|^{2} \\
& =e^{-\pi H(z+u, z+u)}\left|e^{\pi H(z, u)+(\pi / 2) H(u, u)}\right|^{2} \\
& =e^{-\pi H(z, z)-\pi H(z, u)-\pi H(u, z)-\pi H(u, u)}\left|e^{\pi H(z, u)+(\pi / 2) H(u, u)}\right|^{2} \\
& =e^{-\pi H(z, z)} \cdot e^{2 \operatorname{Re}(-\pi H(z, u))-\pi H(u, u)}\left|e^{\pi H(z, u)+(\pi / 2) H(u, u)}\right|^{2} \\
& =e^{-\pi H(z, z)} \\
& =\varphi(z) .
\end{aligned}
$$

Hence $\varphi(z)=\left|e_{u}(z)\right|^{2} \varphi(z+u)$ i.e. $\varphi(z)$ defines a metric on $L(H, \alpha)$. We calculate the curvature.

$$
d^{\prime} \varphi=-\pi\left(\sum_{1 \leq i, j \leq n} h_{i j} \bar{z}_{j} d z_{i}\right) \varphi
$$




$$
\begin{aligned}
d^{\prime \prime} \varphi= & -\pi\left(\sum_{1 \leq i, j \leq n} h_{i j} z_{i} d \bar{z}_{j}\right) \varphi \\
d^{\prime} d^{\prime \prime} \varphi= & -d^{\prime} \varphi \wedge \pi\left(\sum_{1 \leq i, j \leq n} h_{i j} z_{i} d \bar{z}_{j}\right) \\
& -\varphi \cdot \pi \sum_{1 \leq i, j \leq n} h_{i j} d z_{i} \wedge d \bar{z}_{j} \\
\Theta= & \varphi^{-2}\left(-\varphi d^{\prime} d^{\prime \prime} \varphi+d^{\prime} \varphi \wedge d^{\prime \prime} \varphi\right) \\
= & \pi \sum_{1 \leq i, j \leq n} h_{i j} d z_{i} \wedge d \bar{z}_{j}
\end{aligned}
$$

where $H=\left(h_{i j}\right)$.

Proposition (1.10) Let $E$ be a vector bundle. If $E$ is positive (resp. non-negative), then so is any quotient bundle $F$ of $E$.

Proof. We prove the dual assertion. Let $E$ be negative (resp. nonpositive) and $F$ be a sub-vector bundle, then $F$ is negative (resp. nonpositive) by considering the induced metric. For the details see Griffiths [4] p. 197.

Proposition (1.11) Let $E$ and $F$ be vector bundles

(i) $E>0$ and $F>0$ if and only if $E \oplus F>0$

(i) $E$ and $F$ are positive in the sense of Nakano if and only if $E \oplus F$ is positive in the sense of Nakano.

(ii) If $E>0$ and $F \geq 0$, then $E \otimes F>0$.

(ii)' If $E$ is positive in the sense of Nakano and $F$ is non-negative in the sense of Nakano, then $E \otimes F$ is positive in the sense of Nakano.

Proof. (i) is an easy consequence of Proposition (1.10) and the definitions. The proof of (i) ${ }^{\prime}$ is similar.

Let $h_{E}$ and $h_{F}$ be metrics on $E$ and on $F$, respectively. The pairing

$$
\begin{aligned}
(E \otimes F) \times(E \otimes F) \stackrel{h_{E} \otimes h_{F}}{\longrightarrow} C \underset{C}{\otimes} C & \simeq C \\
(a \otimes b, c \otimes d) & \longrightarrow h_{E}(a, c) h_{F}(b, d)
\end{aligned}
$$

defines a metric on $E \otimes F$. Calculation shows that the curvature of $h_{E \otimes F}$ is $\Theta_{E} \otimes I_{s}+I_{r} \otimes \Theta_{F}$ where $r$ (resp. $s$ ) is the rank of $E$ (resp. $F$ ). (ii) and (ii)' follow from what we have shown (See Griffiths [4] p. 209).

DEFINITION (1.12) A vector bundle $E$ is negative in the sense of Grauert if there exists a relatively compact and strongly pseudoconvex 
neighbourhood of the zero-section of $E$. A vector bundle is positive in the sense of Grauert if $\check{E}$ is negative in the sense of Grauert.

A vector bundle $E$ over $V$ is said to be ample if, for any coherent sheaf $F$ on $V$, we have

$H^{i}\left(V, S^{n}(E) \otimes F\right)=0$ for sufficiently large $n$ and $i>0$.

Proposition (1.13). A vector bundle $E$ is ample if and only if the tautological bundle is ample.

Proof. See Hartshorne [5] p. 69.

Proposition (1.14). A vector bundle $E$ is positive in the sense of Grauert if and only if $E$ is ample.

Proof. If $E$ is positive in the sense of Grauert, then $E$ is ample by Grauert [3] p. 344 Hilfssatz 1.

If $E$ is ample, then $E$ is positive in the sense of Grauert by Hartshorne [5] p. 72 Proposition (3.5).

We recall a well known

Proposition (1.15) A line bundle is positive if and only if it is ample.

Proposition (1.16) A positive vector bundle $E$ is ample.

Proof. We deduce the Proposition from Proposition (1.15) and from the direct calculation of the curvature form of the metric on $\mathrm{O}_{p(E)}(1)$ induced by the metric on $E$ (See Griffiths [4]).

Another proof. We shall show that $E$ is negative in the sense of Grauert. Consider the function $\varphi$ on $\check{E}$ defined as in Remark (1.3). Then $\{Q \in \check{E} \mid \varphi(Q)<1\}$ is a relatively compact strongly pseudoconvex neighbourhood of the zero-section. Hence $E$ is negative in the sense of Grauert. It follows that $E$ is ample by Proposition (1.14).

THEOREM (1.17) (Andreotti and Grauert [1], p. 257)

Let $E$ be a vector bundle. If $\Theta_{\xi}(\quad)$ is non-degenerate at any point $P$ of $V$ for any $\xi \neq 0$, then the number $i$ of the negative eigen values of $\Theta_{\xi}(\quad)$ is independent of $P$ and $\xi$ if $\xi \neq 0$ and we have

$$
H^{q}\left(V, S^{m}(E)\right)=0 \text { for sufficiently large } m \text { if } i \neq q \text {. }
$$


Sketch of the proof. First, Andreotti and Grauert show that there exists a filtration in $H^{q}\left(E, O_{E}\right)$ such that the associated graded module $G H^{q}\left(E, O_{E}\right)$ is isomorphic to $\oplus_{n \geq 0} H^{q}\left(V, S^{n}(E)\right)$. Secondely, by Remark (1.3), considering the functions $\varphi$ and $e^{-c \varphi}$ with $c \gg 0$, we deduce that $E$ is strongly $(q+1)$-pseudoconvex and strongly $(n+r-q)$-pseudoconcave. $H^{q}\left(E, O_{E}\right)$ is finite dimensional if $q \neq i$. Now the theorem follows from what we have seen. For the details see Andreotti and Grauert [1]. (cf. Theorem (4.1.1), Theorem (4.2) and Theorem (4.3.1))

(1.18) Let $\varphi=\left\{\varphi_{\alpha}\right\}$ and $\psi=\left\{\psi_{\alpha}\right\}$ be $E$-valued differential forms. We define the inner product of $\varphi$ and $\psi$ by

$$
(\varphi, \psi)=\int_{V} \sum_{\rho, \sigma} h_{\alpha \sigma}^{\rho} \varphi_{\alpha \rho} \wedge \overline{* \psi}_{\alpha \sigma}
$$

This defines a positive definite hermitian metric on the space of $E$-valued differential forms. The adjoint operators of $D$ and $d^{\prime \prime}$ exist

$$
D \leftrightarrow \delta^{\prime}, \quad d^{\prime \prime} \leftrightarrow \vartheta
$$

where $\vartheta_{\alpha}=\delta^{\prime \prime} \varphi_{\alpha}-* \theta_{\alpha} \wedge * \varphi_{\alpha}, \delta^{\prime}=-* d^{\prime \prime} *$ and $\delta^{\prime \prime}=-* d^{\prime} *$. Then, $\left(D^{\prime \prime} D^{\prime}\right.$ $\left.+D^{\prime} D^{\prime \prime}\right) \varphi_{\alpha}=\Theta_{\alpha} \varphi_{\alpha}$. We set $\square=D^{\prime \prime} \vartheta+\vartheta D^{\prime \prime}$. An $E$-valued form $\varphi$ is called harmonic if $\square \varphi=0$ or equivalently $D^{\prime \prime} \varphi=0, \vartheta \varphi=0$.

$$
H^{q}\left(V, \Omega^{p}(E)\right) \simeq\{E \text {-valued harmonic }(p, q) \text { forms }\} \text {. }
$$

LEMma (1.19) (Nakano). Let $\varphi$ be an E-valued harmonic form. Then

$$
-\frac{\sqrt{-1}}{2}(\Lambda \Theta \wedge \varphi, \varphi) \geq 0
$$

Proof.

$$
\begin{aligned}
-\frac{\sqrt{-1}}{2}(\Lambda \Theta \wedge \varphi, \varphi)= & -\frac{\sqrt{-1}}{2}\left(\Lambda\left(D^{\prime \prime} D^{\prime}+D^{\prime} D^{\prime \prime}\right) \varphi, \varphi\right) \\
= & -\frac{\sqrt{-1}}{2}\left(\Lambda D^{\prime \prime} D^{\prime} \varphi, \varphi\right) \\
= & -\frac{\sqrt{-1}}{2}\left(\left(-\sqrt{-1} \delta^{\prime}+d^{\prime \prime} \Lambda\right) D^{\prime} \varphi, \varphi\right), \\
& \quad \text { since } \Lambda d^{\prime \prime}-d^{\prime \prime} \Lambda=-\sqrt{-1} \delta^{\prime} \quad \text { (See Weil [19]). }
\end{aligned}
$$




$$
\begin{aligned}
& =-\frac{\sqrt{-1}}{2}\left(\left(-\sqrt{-1} \delta^{\prime} D^{\prime} \varphi, \varphi\right)+\left(d^{\prime \prime} \Lambda D^{\prime} \varphi, \varphi\right)\right) \\
& =-\frac{\sqrt{-1}}{2}\left(\left(-\sqrt{-1} \delta^{\prime} D \varphi, \varphi\right)+\left(D^{\prime \prime} \Lambda D^{\prime} \varphi, \varphi\right)\right) \\
& =-\frac{\sqrt{-1}}{2}\left(\left(-\sqrt{-1} \delta^{\prime} D \varphi, \varphi\right)+\left(\Lambda D^{\prime} \varphi, \varphi\right)\right) \\
& =-\frac{\sqrt{-1}}{2}\left(\left(-\sqrt{-1} \delta^{\prime} D \varphi, \varphi\right)\right. \\
& =-\frac{1}{2}\left(\delta^{\prime} D \varphi, \varphi\right) \\
& =-\frac{1}{2}(D \varphi, D \varphi) \leq 0
\end{aligned}
$$

q.e.d.

\section{$\$ 2$ Positive vector bundles over a compact Riemann surface}

LEMMA (2.1) Let $V$ be a manifold or an algebraic variety defined over an algebraically closed field $k$. Let $E_{1}$ and $E_{2}$ be vector bundles on $V$. Let $E_{\xi}$ be the extension of $E_{2}$ by $E_{1}$;

$$
0 \rightarrow E_{1} \rightarrow E_{\xi} \rightarrow E_{2} \rightarrow 0
$$

determined by an element $\xi \in H^{1}\left(V, \operatorname{Hom}\left(E_{2}, E_{1}\right)\right)$. Then $E_{\xi} \simeq E_{\lambda \xi}$ for any $0 \neq \lambda \in k$.

Proof. Consider the commutative diagram

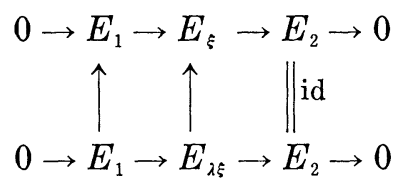

where the vertical arrow on the left is the multiplication by $\lambda$.

LEMMA (2.2) Let $V$ be a non-singular projective algebraic variety defined over $C$. Let $E_{1}$ and $E_{2}$ be vector bundles on $V$. If $E_{1}$ and $E_{2}$ are positive in the sense of Nakano, then an extension of $E_{2}$ by $E_{1}$ is positive in the sense of Nakano.

Proof. Let $E$ be defined by $v \in H^{1}\left(V, \operatorname{Hom}\left(E_{2}, E_{1}\right)\right)$. Take a sufficiently fine open covering $\left\{U_{\alpha}\right\}$ of $V$ so that the extension $E_{V}$ is given by patching $E_{1 \mid U_{\alpha}} \oplus E_{2 \mid U_{\alpha}}$ and $E_{1 \mid U_{\beta}} \otimes E_{2 \mid U_{\beta}}$ on $U_{\alpha} \cap U_{\beta}$ by $\left(\begin{array}{ll}I & a_{\beta \alpha} \\ 0 & I\end{array}\right)$ where $a_{\beta \alpha} \in$ $\Gamma\left(U_{\alpha} \cap U_{\beta}, \operatorname{Hom}\left(E_{2}, E_{1}\right)\right)$ and $\left(x_{\alpha}, y_{\alpha}\right) \in E_{1 \mid U_{\alpha}} \oplus E_{2 \mid U_{\alpha}}$ and $\left(x_{\beta}, y_{\beta}\right) \in E_{1 \mid U_{\beta}} \oplus E_{2 \mid U_{\beta}}$ 
are identified if $\left(x_{\alpha}+a_{\beta \alpha} y_{\alpha}, y_{\alpha}\right)=\left(x_{\beta}, y_{\beta}\right)$. Then the extension $E_{\lambda v}$ is defined by replacing $\left(\begin{array}{ll}I & a_{\beta \alpha} \\ 0 & I\end{array}\right)$ by $\left(\begin{array}{cc}I & \lambda a_{\beta \alpha} \\ 0 & I\end{array}\right)$. Since an extension is differentiably trivial, there exists a $C^{\infty}$ homomorphism $b_{\alpha}: E_{2 \mid U_{\alpha}} \rightarrow E_{1 \mid U_{\alpha}}$ for each $\alpha$ such that $a_{\beta \alpha}=b_{\alpha}-b_{\beta}$ on $U_{\alpha} \cap U_{\beta}$;

$$
\begin{aligned}
& E_{1 \mid U_{\alpha}} \oplus E_{2 \mid U_{\alpha}} \stackrel{\left(\begin{array}{cc}
I & \lambda a_{\beta \alpha} \\
0 & I
\end{array}\right)}{\longrightarrow} E_{1 \mid U_{\beta}} \oplus E_{2 \mid U_{\beta}} \\
& \downarrow\left(\begin{array}{cc}
I & \lambda b_{\alpha} \\
0 & I
\end{array}\right) \quad \bullet \downarrow \downarrow\left(\begin{array}{cc}
I & \lambda b_{\beta} \\
0 & I
\end{array}\right) \\
& E_{1 \mid U_{\alpha}} \oplus E_{2 \mid U_{\alpha}} \underset{\text { id }}{\sim} E_{1 \mid U_{\beta}} \oplus E_{2 \mid U_{\beta}}
\end{aligned}
$$

Let $h_{E_{1}}$ and $h_{E_{2}}$ be hermitian metrics on $E_{1}$ and $E_{2}$ such that their curvature forms,

$$
\sum_{\substack{1 \leq p, \tau, \sigma \leq r \\ 1 \leq i, j \leq n}} h_{E_{1}}{ }_{\tau}^{\rho} \Theta_{E_{1}}{ }^{\tau}{ }_{\sigma i j} \xi_{1}^{(\rho, i)} \overline{\xi_{2}^{(\sigma, j)}}
$$

and

$$
\sum_{\substack{1 \leq \rho, \tau, \sigma \leq s \\ 1 \leq i, j \leq n}} h_{E_{2}}{ }_{\tau}^{\rho} \Theta_{E_{2}}{ }_{\sigma i j}^{\tau} \xi_{2}^{(\rho, i)} \overline{\xi_{2}^{(\sigma, j)}}
$$

are positive definite. We set

$$
h_{E_{\lambda v} \alpha}={ }^{t}\left(\begin{array}{cc}
I & -\lambda b_{\alpha} \\
0 & I
\end{array}\right)\left(\begin{array}{cc}
h_{1 \alpha} & 0 \\
0 & h_{2 \alpha}
\end{array}\right)\left(\begin{array}{cc}
I & -\lambda b_{\alpha} \\
0 & I
\end{array}\right) .
$$

$\left\{h_{E_{\lambda v} \alpha}\right\}$ defines a hermitian metric on $E_{\lambda V}$. If we set $H_{\lambda}(\xi)=$

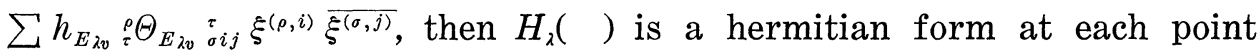
$P$ of $V$. We fix a point $P$. Then there exists a number $c_{p}>0$ and an open neighbourhood $U_{p}$ of $P$ such that $H_{\lambda}$ is positive definite at any point $Q \in U_{p}$ if $|\lambda|<c_{p}$, since $H_{0}$ is positive definite at $P$. Since $V$ is compact, there exists a number $c>0$ such that $H_{\lambda}$ is positive definite at any point if $|\lambda|<c$. Now the lemma follows from lemma (2.1).

LEMMA (2.3) Let $V$ be a non-singular projective algebraic variety defined over $C$. Let $E$ be a vector bundle of rank $r$ on $V$. Let $\left\{U_{\alpha}\right\}$ be an open covering of $V$ such that $E_{\mid U_{\alpha}}$ is trivial. Assume that the transition matrices $g_{\beta \alpha}$ can be written in the form $g_{\beta \alpha}=f_{\beta \alpha} \cdot U_{\beta \alpha}$ where $f_{\beta \alpha}$ is a scalar function and $U_{\beta \alpha}$ is a unitary matrix on $U_{\alpha} \cap U_{\beta}$. If $\operatorname{det} E$ is positive, then $E$ is positive in the sense of Nakano. In particular $E$ is positive and ample. 
Proof. Let $\left\{h_{\alpha}\right\}$ be a hermitian metric on $\operatorname{det} E$ such that its curvature form is positive definite. From the definition $\mid \operatorname{det} g_{\beta \alpha}{ }^{2} h_{\alpha}=h_{\beta}$ on $U_{\alpha} \cap U_{\beta}$ i.e. $\left|f_{\beta \alpha}\right|^{2 r} \cdot h_{\alpha}=h_{\beta}$. Hence $\mid f_{\beta \alpha}{ }^{2} h_{\alpha}^{1 / r}=h_{\beta}^{1 / r}$. Consider the matrix $h_{\alpha}^{1 / r} I_{r}$. This is a positive definite hermitian metric on $E \mid U_{\alpha}$. On the other hand, we have

$$
\begin{aligned}
{ }^{t} \bar{g}_{\beta \alpha} h_{\alpha}^{1 / r} I_{r} g_{\beta \alpha} & =\bar{f}_{\beta \alpha} \cdot{ }^{t} \bar{U}_{\beta \alpha} \cdot h_{\alpha}^{1 / r} I_{r} \cdot f_{\beta \alpha} \cdot U_{\beta \alpha} \\
& =\left|f_{\beta \alpha}\right|^{2} h_{\alpha}^{1 / r} t \bar{U}_{\beta \alpha} U_{\beta \alpha} \\
& =\left|f_{\beta \alpha}\right| h_{\alpha}^{1 / r} I_{r} \\
& =h_{\beta}^{1 / r} I_{r} .
\end{aligned}
$$

Hence $\left\{h_{\alpha}^{\mathrm{t} / r} I_{r}\right\}$ defines a hermitian metric on $E$. Let $\Theta$ be the curvature form of $\left\{h_{\alpha}\right\}$. Then the curvature form of $\left\{h_{\alpha}^{1 / r} I_{\alpha}\right\}$ is given by $(1 / r) \Theta I_{r}$. Hence $E$ is positive in the sense of Nakano.

q.e.d.

(2.4) We need some results of Narasimhan and Seshadri [11].

Let $S$ be a compact Riemann surface of genus $g \geqq 2$. Let $\pi$ be a discrete group acting effectively, properly and holomorphically on the unit disc $T$ such that $T / \pi \simeq S$ and such that the projection $P: T \rightarrow S$ is unramified except at only one point $x_{0}$ and ramified with order $n$ at $x_{0}$. Such a group $\pi$ always exists. Let $\rho: \pi \rightarrow G L(n ; C)$ be a representation. Then $\pi$ operates on the trivial bundle $T \times \boldsymbol{C}^{n}$ by $(y, v) \mapsto(y, \rho(\gamma) v)$, $y \in T, v \in C^{n}, \gamma \in \pi$. We denote by $E_{\pi}(\rho)$ this vector bundle carrying the action of $\pi$. We denote by $P_{*}^{\pi}(E(\rho))$ the subsheaf of $P_{*}(E(\rho))$ consisting of elements invariant under the action of $\pi$. Then $P_{*}^{\pi}(E(\rho))$ is a vector bundle of rank $n$ on $S$. We call $E_{\pi}(\rho)$ the $\pi$-bundle associated to $\rho$. The vector bundle $P_{*}^{\pi}(E(\rho))$ is called the vector bundle arising from the representation $\rho$ of $\pi$.

Let $y_{0} \in p^{-1}\left(x_{0}\right)$ and $\pi_{y_{0}}$ be the isotropy group of $\pi$ at $y_{0}$. Let $z$ be a coordinate system around $y_{0}$ such that the action of $\pi_{y_{0}}$ is multiplication by $\zeta^{k}$ where $\zeta$ is a primitive $n^{t h}$ root of unity. Let $\gamma_{0}$ be the generator of $\pi_{y_{0}}$ corresponding to multiplication by $\zeta$. Let $\tau$ be a character of $\pi_{y_{0}}$. If $\tau\left(\gamma_{0}\right)=\zeta^{s}, 0 \leq s<n$, then the integer $s$ is independent of $s$ and $z$. The integer $s$ is called the associated integer to $\tau$.

A homomorphism $\rho: \pi \rightarrow U(n, C)$ is a representation of type $\tau$, by definition, if for every $\gamma \in \pi_{y_{0}}$, we have $\rho(\gamma)=\tau(\gamma) I_{n}$.

A vector bundle $E$ of rank $r$ over a compact Riemann surface is said to be stable if 


$$
\frac{\text { degree } E}{\operatorname{rank} E}<\frac{\text { degree } F}{\operatorname{rank} F}
$$

for any quotient bundle $F$ of $E$.

THEOREM (2.4.1) (Narasimhan and Seshadri [11]) $A$ vector bundle $F$ of rank $n$ and degree $-n<q \leq 0$ over a compact Riemann surface of genus $\geq 2$ is stable if and only if $F$ is isomorphic to $P_{*}^{\pi}(E(\rho))$ where $\rho$ is an irreducible unitary representation of type $\tau$ and the associated integer to $\tau$ is $q$.

COROLLARY (2.4.2). Let $E$ be a stable vector bundle of rank $r$ over a compact Riemann surface $R$ of genus $\geq 2$, then there exists an open covering $\left\{U_{\alpha}\right\}$ of $R$ such that $E_{\mid U_{\alpha}}$ is trivial for each $\alpha$ and such that the transition matrices can be written in the form;

Scalar function $\times$ unitary matrix.

Proof of the Corollary. If the transition matrices are of the desired form, so are the transition matrices of $E \otimes L$ for any line bundle $L$ on $R$. Hence we may assume $-n<\operatorname{deg} E \leq 0$. In this case the corollary is an easy consequence of the Theorem and Narasimhan and Seshadri [11] Remark 6.2. p. 550.

LEMMA (2.5) A stable bundle of positive degree over a compact Riemann surface of genus $\geq 2$ is positive in the sense of Nakano.

Proof. The Lemma is an easy consequence of Lemma (2.2) and Corollary (2.4.2).

THEOREM (2.6) Let $R$ be a compact Riemann surface of genus $g$. Let $E$ be a vector bundle of rank $r$ over $R$. Then the following are equivalent.

(i) $E$ is positive in the sense of Nakano.

(ii) $E$ is positive.

(iii) $E$ is ample.

(iv) The degree of every quotient bundle of $E$ (including $E$ itself) is positive.

Proof. The equivalence of (i) and (ii) follows from Lemma (1.5). (ii) $\Rightarrow$ (iii) follows from Proposition (1.16). Since any quotient bundle of an ample vector bundle is ample, (iii) $\Rightarrow$ (iv). Hence it is sufficient 
to show that (iv) $\Rightarrow$ (i). Let $E$ be a vector bundle on $R$ such that the degree of any quotient bundle is positive. If $g=0, E$ is the direct sum of line bundles. Hence the assertion follows from Proposition (1.11). If $g=1$. The assertion was proved in Umemura [18]. We may assume $g \geq 2$. We proceed by induction on the rank $r$ of $E$.

If $r=1$, the assertion (iv) $\Rightarrow$ (i) follows from Proposition (1.15) and the Riemann-Roch theorem.

Now we assume that the assertion (iv) $\Rightarrow$ (i) is proved for rank strictly less than $r$. Suppose that $E$ contains a subbundle $E_{1}$ which is positive in the sense of Nakano:

$$
0 \rightarrow E_{1} \rightarrow E \rightarrow E_{2} \rightarrow 0 \text {. }
$$

$E_{2}$ is positive by the inductive hypothesis since every quotient bundle of $E_{2}$ has positive degree. Hence by Lemma (2.2), $E$ is positive in the sense of Nakano. If $E$ does not contain a subbundle which is positive in the sense of Nakano, then $E$ is stable. In fact, let $F$ be a subbundle of $E$. We prove that the degree $F$ is $\leq 0$. We use induction on the rank $s$ of $F$. If $s=1$, then the degree $F \leq 0$ since otherwise $F$ would be positive in the sense of Nakano. Now we suppose that the degree of a subbundle is $\leq 0$ if its rank is less than $s$. Let $F$ be a subbundle of rank $s$. By the inductive hypothesis every subbundle of $F$ has degree $\leq 0$. Hence if the degree of $F$ were positive, every quotient bundle of $F$ would be positive. By the inductive hypothesis $F$ would be positive in the sense of Nakano. Hence we may assume $E$ to be stable. Since the degree of $E$ is positive, $E$ is positive in the sense of Nakano by Lemma (3.5).

Remark (2.7). Hartshorne proved the equivalence of (iii) and (iv) (cf. Hartshorne [6]).

\section{§3. Some positive vector bundles of rank 2 over an algebraic surface.*)}

(3.1) We recall the results of Takemoto [14] and [15]. Let $S$ be a nonsingular projective surface defined over $C$. Let $E$ be a vector bundle of rank 2 over $S$. Let $H$ be an ample line bundle over $S$.

Definition (3.1.1) $E$ is said to be $H$-stable (resp. $H$-semi-stable) if for any successive blowing ups $\pi: S^{\prime} \rightarrow S$ and for any sub line bundle $F$ $\frac{\text { of } \pi^{*} E \text {, we have }}{* \text { See }[20]}$ 
$(\operatorname{det} E, H) / 2>\left(F, \pi^{*} H\right)$

$($ resp. $\geq$ )

where (, ) denotes the intersection number.

Proposition (3.1.2) An $H$-stable bundle is simple i.e. $H^{0}(S$, End $(E))$ $=C$.

THEOREM (3.1.3) The set of all H-stable vector bundles of rank 2 with fixed numerical Chern class is bounded i.e. there exists a scheme $T$ of finite type over $C$ and a vector bundle $E$ on $T \times S$ such that, for any $H$-stable vector bundle $F$ of rank 2 with the fixed numerical Chern class, there exists a closed point $t \in T$ with $F \simeq E_{\mid t \times s}$.

THEOREM (3.1.4) Let $H_{1}, H_{2}$ be ample line bundles on S. Assume that $S$ is relatively minimal and $N(E)=c_{1}^{2}-4 c_{2} \geq 0$ where $c_{i}$ is $i$-th Chern class of $E$. Then $E$ is $H_{1}$-stable if and only if $E$ is $H_{2}$-stable.

Remark (3.1.5) If $N(E)<0$, the $H$-stability depends on the choice of an ample line bundle $H$. (See Takemoto [14]. See also Example (3.3)). By the Riemann-Roch theorem, $N(E) \leq 0$ on an abelian surface and $N(E) \leq-2$ on $P^{2}$.

Proposition (3.1.6) Let $G$ be a finite solvable group. Let $G$ operate on $S$ holomorphically so that the projection $\pi: S \rightarrow S / G$ is unramified. Let $H$ be an ample line bundle on $S / G$. Let $E$ be an $H$-semi-stable bundle on $S / G$. Then $\pi^{*} E$ is $\pi^{*} H$-semi-stable.

DEFINITION (3.1.7) A non-singular projective surface $S$ is said to be hyperelliptic if the first Betti number of $S$ is 2 and if there exist an elliptic curve $\Delta$ and a smooth morphism $\pi: S \rightarrow \Delta$ such that every fibre is an elliptic curve.

THEOREM (3.2) Let $S$ be either an abelian variety of dimension 2, a geometrically ruled surface or a hyperelliptic surface. Let $E$ be a vector bundle of rank 2 over $S$. Let $H$ be an ample line bundle on $S$. If $E$ is $H$-stable with $N(E) \geq 0$ and $c_{1}>0$, then $E$ is positive in the sense of Nakano.

Proof. Case I. $S=A$ is an abelian variety. In this case, we prove a slightly more general assertion: If $E$ is $H$-semi-stable with 
$N(E) \geq 0$, and $c_{1}>0$, then $E$ is positive in the sense of Nakano. If $E$ is simple, then by Oda [12], there exists an isogeny $p: A^{\prime} \rightarrow A$ of degree 2 and a line bundle $L$ on $A^{\prime}$ such that $p_{*} L \simeq E$. We have $p^{*} p_{*} L \simeq$ $\underset{x \in \operatorname{Ker} p}{\oplus} T_{x}^{*} L$ by Oda [12] where $T_{x}$ denotes translation by $x$. Since $p$ is finite and $\operatorname{det} p_{*} L$ is ample by the Nakai criterion and the hypothesis, $\operatorname{det}\left(p^{*} p_{*} L\right)$ is ample. Since $L$ and $T_{x}^{*} L$ are numerically equivalent, $\operatorname{det}\left(p^{*} p_{*} L\right)$ is numerically equivalent to $L^{\otimes 2}$. It follows that $L^{\otimes 2}$ is ample by the Nakai criterion hence $L$ is ample or equivalently positive in the sense of Nakano. The direct image $p_{*} L$ is positive in the sense of Nakano.

If $E$ is not simple, then, by Proposition (5.2), Takemoto [14], ${ }^{*} E$ is written in the form $E^{\prime} \otimes M$ where $E^{\prime}$ is an extension:

$$
0 \rightarrow O \rightarrow E^{\prime} \rightarrow O \rightarrow 0
$$

and $M$ a line bundle on $A$. Hence

$$
\begin{aligned}
c(E) & =(1+M t)(1+M t) \\
& =1+M^{\otimes 2} t+\left(M^{2}\right) t^{2} .
\end{aligned}
$$

From our hypothesis $M^{\otimes 2}$ is positive, hence ample by the Nakai criterion. It follows that $M$ is ample. Since $M$ is a line bundle, $M$ is positive in the sense of Nakano by Proposition (1.15). By Lemma (2.2) the extension $0 \rightarrow M \rightarrow E^{\prime} \otimes M \rightarrow M \rightarrow 0$ is positive in the sense of Nakano.

Case II. Geometrically ruled surfaces. Let $p: S=P(V) \rightarrow C$ be a geometrically ruled surface over a curve $C$ of genus $g$. We know from the hypothesis and Takemoto [14] that there exist a stable vector bundle $F$ of rank 2 over $C$ and a line bundle $L$ on $P(V)$ such that $E \simeq L \otimes p^{*} F$.

If $g=0$, we have nothing to prove, since there is no stable bundle of rank 2 on $C$.

If $g=1$, there exist an isogeny $\pi: C^{\prime} \rightarrow C$ of elliptic curves and a line bundle $L^{\prime}$ on $C^{\prime}$ such that $\pi_{*} L^{\prime} \simeq F$ by Oda [12]. Consider the diagram :

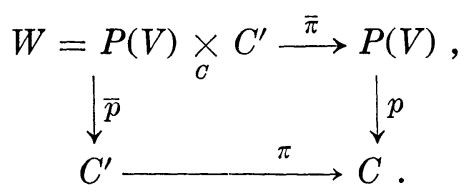

\footnotetext{
*) See (Added in proof) p. 127.
} 
Since $\pi$ is affine, we have $p^{*} F \simeq \bar{\pi}_{*} \bar{p}^{*} L^{\prime}$. Hence $\bar{\pi}_{*}\left(\bar{p}^{*} L^{\prime} \otimes \bar{\pi}^{*} L\right) \simeq E$. Let $N$ be the kernel of $\pi$. Then $W$ is a Galois covering of $P(V)$ with Galois group $N$. By Takemoto [15], we have $\bar{\pi}^{*} E \simeq \bigoplus_{g \in N} T_{g}^{*}\left(\bar{p}^{*} L^{\prime} \otimes \bar{\pi}^{*} L\right)$ where $T_{g}$ denotes the operation of $g$ on $W$. As in case I, we know $\operatorname{det}\left(\bar{p}^{*} L^{\prime} \otimes \bar{\pi}^{*} L \oplus T_{g}^{*}\left(\bar{p}^{*} L^{\prime} \otimes \bar{\pi}^{*} L\right)\right)$ is ample with $e \neq g \in N$. On the other hand, $T_{g}^{*}\left(\bar{p}^{*} L^{\prime} \otimes \bar{\pi}^{*} L\right) \simeq T_{g}^{*}\left(\bar{p}^{*} L^{\prime}\right) \otimes T_{g}^{*}\left(\bar{\pi}^{*} L\right)=\bar{p}^{*}\left(T_{p}^{*} L^{\prime}\right) \otimes \bar{\pi}^{*} L$. Since $T_{g}^{*} L^{\prime}$ and $L^{\prime}$ are numerically equivalent, we proved that $\operatorname{det}\left(\bar{p}^{*} L^{\prime} \otimes \bar{\pi}^{*} L \oplus\right.$ $\left.T_{g}^{*}\left(\bar{p}^{*} L^{\prime} \otimes \bar{\pi}^{*} L\right)\right)$ is numerically equivalent to $\left(\bar{p}^{*} L^{\prime} \otimes \bar{\pi}^{*} L\right)^{\otimes 2}$. Hence $\bar{p}^{*} L^{\prime} \otimes \bar{\pi}^{*} L$ is ample by the Nakai criterion and the direct image $\bar{\pi}_{*}\left(\bar{p}^{*} L^{\prime} \otimes \bar{\pi}^{*} L\right)=E$ is positive in the sense of Nakano.

If $g \geq 2$, then by Corollary (2.4.2) the transition matrices of $F$ can be written in the form:

Scalar function $\times$ unitary matrix.

Hence $\pi^{*} F$ and $\pi^{*} F \otimes L$ have the same property. By Lemma (2.3), the Theorem is proved for geometrically ruled surfaces.

Case III. Hyperelliptic surface. In this case, there exists an abelian variety $A$ and a finite abelian group $G$ such that $G$ operates on $A, A / G$ is isomorphic to $S$ and the projection $\pi: A / G \rightarrow S$ is unramified. By Proposition (3.1.6), $\pi^{*} E$ is $\pi^{*} H$-semi-stable with $N\left(\pi^{*} E\right)=0$. By what we have proved in case I, $\pi^{*} E$ is positive in the sense of Nakano. Hence $\pi_{*} \pi^{*} E$ is positive in the sense of Nakano. Since $E$ is a direct summand of $\pi_{*} \pi^{*} E, E$ is positive in the sense of Nakano by Proposition (1.11).

q.e.d.

ExAMPLE (3.3) Let $E_{1}, E_{2}$ be elliptic curves. Let $M_{i}^{\prime}$ be a line bundle of degree 1 on $E_{i}, i=1,2$. We put $A=E_{1} \times E_{2}$ and $p_{i}^{*} M_{i}^{\prime}=M_{i}$ for $i=1,2$ where $p_{i}$ is the projection $p_{i}: A=E_{1} \times E_{2} \rightarrow E_{i}$. We set $L_{1}=$ $M_{1}^{\otimes n_{1}} \otimes M_{2}^{\otimes n_{2}}, L_{2}=M_{1}^{\otimes m_{1}} \otimes M_{2}^{\otimes m_{2}}$. We define a vector bundle $E$ of rank 2 by the exact sequence

$$
0 \rightarrow L_{1} \rightarrow E \rightarrow L_{2} \rightarrow 0
$$

Then

$$
\begin{aligned}
& c_{1}(E)=M_{1}^{\otimes\left(n_{1}+m_{1}\right)} \otimes M_{2}^{\otimes\left(n_{2}+m_{2}\right)} \\
& c_{1}(E)=n_{2} m_{1}+n_{1} m_{2} .
\end{aligned}
$$

If $n_{1}-m_{1}$ and $n_{2}-m_{2}$ are coprime, $n_{1}>m_{1}, n_{2}<m_{2}$ and if we take a non-trivial extension, then, by Takemoto [14] p. $41, E$ is $H$-stable for a 
certain ample line bundle $H$ on $A$. But there exists an ample line bundle $H^{\prime}$ such that $E$ is not $H^{\prime}$-stable. In fact we have $c_{1}^{2}-4 c_{2}<0$. It is easy to see that $c_{1}>0, c_{2}>0, c_{1}^{2}-c_{2}>0$ if and only if $n_{1}+m_{1}$ $>0, \dot{n}_{2}+m_{2}>0, n_{2} m_{1}+n_{1} m_{2}>0,2 n_{1} n_{2}+n_{1} m_{2}+m_{1} n_{2}+2 m_{1} m_{2}>0$. Hence if we take $n_{1}=1000, n_{2}=0, m_{1}=-1, m_{2}=1$, then $E$ is $H$-stable with $c_{1}>0, c_{2}>0, c_{1}^{2}-c_{2}>0$ and $c_{1}^{2}-4 c_{2}<0$. Since $L_{2}$ is not ample, $E$ is not ample. (see Remark (3.1.5)).

Remark (3.4) Theorem (3.2) is an analogue of Lemma (2.5). It is natural to ask:

Problem (3.4.1) Let $S$ be a non-singular projective surface defined over $C$. Let $H$ be an ample line bundle. Let $E$ be a vector bundle of rank 2 on $S$. Assume that $E$ is $H$-stable with $N(E)=c_{1}^{2}-4 c_{2} \geq 0$, $c_{1}>0, c_{2}>0$. Then is $E$ ample?

(3.4.2) The answer may be negative in characteristic $p>0$, because in characteristic $p>0$, there exists a curve $C$ of genus $g \geq 2$ such that there exists a stable bundle of positive degree on $C$ which is not ample (cf. Hartshorne [6]).

\section{§ 4. Vanishing theorems}

(4.1) The following theorem is well known. We prove it by our own methods.

Theorem (4.1.1) Let $A$ be an abelian variety of dimension $n$ defined over $C$. Let $L=L(H, \alpha)$ be a non-degenerate line bundle over $A$. Then,

$$
H^{i}(A, L)=0 \quad \text { if } i \neq \sharp\{\text { negative eigen values of } H\} \text {. }
$$

Proof. Let $r$ be the number of negative eigen values of $H$. We choose a coordinate system $\left(z_{1}, \cdots, z_{n}\right)$ of the universal covering space $C^{n}$ of $A$ so that $H$ is written in the following form:

$$
\left(\begin{array}{cc}
-I_{r} & 0 \\
0 & \frac{1}{n-r+1} I_{n-r}
\end{array}\right)
$$

We use the metric $\varphi(z)$ defined in Example (1.9). Then the curvature form $\Theta$ is given by 


$$
\begin{aligned}
\Theta= & -\pi\left(d z_{1} \wedge d \bar{z}_{1}+\cdots+d z_{r} \wedge d \bar{z}_{r}\right) \\
& +\frac{\pi}{n-r+1}\left(d z_{r+1} \wedge d \bar{z}_{r+1}+\cdots+d z_{n} \wedge d \bar{z}_{n}\right) .
\end{aligned}
$$

We define a kähler metric on $C^{n}$ hence on $A$ by $\omega=\sqrt{-1} / 2 \sum_{i=1}^{n} d z_{i} \wedge d \bar{z}_{i}$. Now the theory of harmonic integrals (1.18) is applied.

LEMMA (4.1.2) If $\varphi=\varphi_{0} d \bar{z}_{i_{1}} \wedge \cdots \wedge d \bar{z}_{i_{q}}$ is a $(0, q)$-form with coefficients in $L$, then

$$
-\frac{\sqrt{-1}}{2}(\Lambda \Theta \wedge \varphi, \varphi) \geq \pi\left(r-q-\frac{n-r}{n-r+1}\right)(\varphi, \varphi) .
$$

Proof of the lemma. We compare integrands. We may assume that $\varphi=d \bar{z}_{i_{1}} \wedge \cdots \wedge d \bar{z}_{i_{q}}$ since the question is local.

$$
\begin{gathered}
-\Theta \wedge \varphi=\pi\left(\left(d z_{1} \wedge d \bar{z}_{1}+\cdots+d z_{r} \wedge d \bar{z}_{r}\right)-\frac{1}{n-r+1}\left(d z_{r+1} \wedge d \bar{z}_{r+1}\right.\right. \\
\left.\left.+\cdots+d z_{n} \wedge d \bar{z}_{n}\right)\right) \wedge d \bar{z}_{i_{1}} \wedge \cdots \wedge d \bar{z}_{i_{q}} .
\end{gathered}
$$

We use the following notation:

$$
\begin{aligned}
& I=\left\{i_{1}, \cdots, i_{q}\right\}, \quad N=\{1, \cdots, n\}, \quad \omega_{\ell}=d z_{\ell} \wedge d \bar{z}_{\ell} . \\
&-\Theta \wedge \varphi= \pi \sum_{\ell \in N-\{r+1, \cdots, n\}-I} d \bar{z}_{i_{1}} \wedge \cdots \wedge d \bar{z}_{i_{q}} \wedge \omega_{\ell} \\
&-\frac{\pi}{n-r+1} \sum_{\ell \in N-\{1, \cdots, r\}-I} d \bar{z}_{i_{1}} \wedge \cdots \wedge d \bar{z}_{i_{q}} \wedge \omega_{\ell} .
\end{aligned}
$$

Hence

$$
\begin{aligned}
-\frac{\sqrt{-1}}{2} \Lambda \Theta \wedge \varphi= & \pi \sum_{\ell \in N-\{1, \cdots, r\}-I} d \bar{z}_{i_{1}} \wedge \cdots \wedge d \bar{z}_{i_{q}} \\
& -\frac{\pi}{n-r+1} \sum_{\ell \in N-\{r+1, \cdots, n\}-I} d \bar{z}_{i_{1}} \wedge \cdots \wedge d \bar{z}_{i_{q}} .
\end{aligned}
$$

The lemma follows from the inequality

$$
\begin{gathered}
\#\{N-\{r+1, \cdots, n\}-I\}-\frac{1}{n-r+1} \times \#\{N-\{1, \cdots, r\}-I\} \\
\geq\{n-(n-r)-q\}-\frac{1}{n-r+1}=r-q-\frac{n-r}{n-r+1} .
\end{gathered}
$$

q.e.d.

Let $\varphi$ be a harmonic form of type $(0, q)$ with coefficients in $L$. Let $\varphi=\sum_{I} \varphi_{I}$ where $I=\left\{i_{1}, \cdots, i_{q}\right\} \subset N$ and $\varphi_{I}=\psi_{I} d \bar{z}_{i_{1}} \wedge \cdots \wedge d \bar{z}_{i_{q}}$ and the 
summation is taken for all such I's.

$$
\begin{aligned}
0 \geq- & \frac{\sqrt{-1}}{2}(\Lambda \Theta \wedge \varphi, \varphi) \text { by Lemma (1.19) } \\
= & \frac{\sqrt{-1}}{2}\left(\Lambda \Theta \wedge \sum_{I} \varphi_{I}, \sum_{I} \varphi_{I}\right) \\
& \text { since }\left(d \bar{z}_{i} \wedge \cdots \wedge d \bar{z}_{i_{q}}\right) \wedge * \overline{\left(d \bar{z}_{j_{1}} \wedge \cdots \wedge d \bar{z}_{j_{q}}\right)}=0 \\
& \text { if }\left\{i_{1}, \cdots, i_{q}\right\} \neq\left\{j_{1}, \cdots, j_{q}\right\}, \\
= & \sum_{I}-\frac{\sqrt{-1}}{2}\left(\Lambda \Theta \wedge \varphi_{I}, \varphi_{I}\right)
\end{aligned}
$$$$
\text { by lemma (4.1.2) }
$$$$
\geq \pi \sum_{I}\left(\left(r-q-\frac{n-r}{n-r+1}\right) \varphi_{I}, \varphi_{I}\right)
$$$$
=\pi\left(\sum_{I}\left(r-q-\frac{n-r}{n-r+1}\right) \varphi_{I}, \sum_{I} \varphi_{I}\right)
$$$$
=\pi\left(r-q-\frac{n-r}{n-r+1}\right)(\varphi, \varphi) \text {. }
$$

Hence if $r-q-(n-r) /(n-r+1)>0$ i.e. $r>q$, we have $H^{q}(A, E)$ $=0$. The line bundle $\check{L}$, dual to $L(H, \alpha)$ is given by $L\left(-H, \alpha^{-1}\right)$ (cf. Mumford [9]). Hence by Serre duality we have $H^{q}(A, L)=0$ for $q>r$. This completes the proof of the theorem.

q.e.d.

THEOREM (4.2)*) Let $V$ be a projective non-singular variety defined over $C$. Let $L$ be a line bundle on $V$ such that $L^{\otimes m}$ is generated by global sections for large $m$. Let $\varphi: V \rightarrow W \subset P^{N}$ be a morphism defined by $L^{\otimes m}$. If $\operatorname{dim} W=q$, then $H^{i}(V, L)=0$ for $i \leq q-1$.

Proof. Let $H$ be a hyperplane section of $P^{N}$. Then, $L^{\otimes m}=\varphi^{*} H$. Let $h^{\prime}$ be a metric on $H$. Then $h=h^{\prime} \circ \varphi$ is a metric on $L^{\otimes m}$. The curvature form $\Theta_{h}$ of $h$ is, by an easy calculation, given by

$$
\Theta_{h}=J \Theta_{h^{\prime}}{ }^{t} \bar{J}
$$

where $J$ is the Jacobian of $\varphi$ and $\Theta_{h^{\prime}}$ is the curvature form of $h^{\prime}$. Hence $h^{1 / \ell}$ defines a metric on $L$ and its curvature form is $1 / \ell \Theta_{h}$. Let $\tilde{\Theta}$ be the curvature form of $L^{-1}$. We fix a kähler metric $\omega$ on $V$. From what we have seen, at a point $Q \in V$, we may assume that

*) This result is independently proved by C. P. Ramanujam: Remarks on the Kodaira vanishing theorem, Jour. of the Indian Math. Soc. 36 (1972) p. 41-51. 


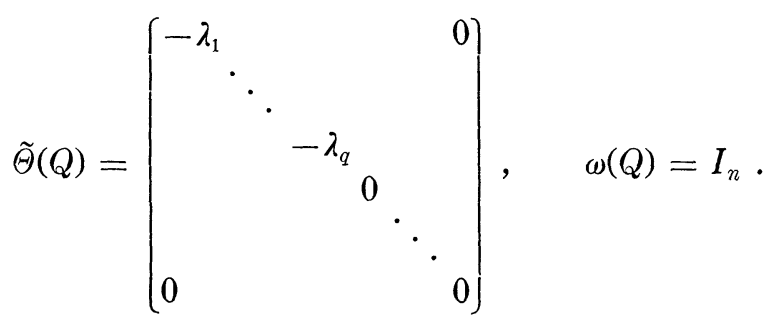

and that there exists a point $Q$ such that $\lambda_{i}>0,1 \leq i \leq q$. i.e. a point where $J$ attains its maximal rank $q$. Let $\varphi$ be a non-zero harmonic $(0, p)$-form with coefficients in $L^{-1}$. Then $\varphi$ is non-zero on a dense open set in $V$. As in the proof of Lemma (4.1.2), the integrand of $-\frac{1}{2}(\Lambda \Theta \wedge \varphi, \varphi)$ is non-negative at any point $Q$ and there exists a point $Q$ where the integrand is positive if $p<q$. Hence if $p<q$, we have $-\sqrt{-1} / 2(\Lambda \Theta \wedge \varphi, \varphi)$ $>0$. On the other hand $-\sqrt{-1} / 2(\Lambda \Theta \wedge \varphi, \varphi) \leq 0$ by Lemma (1.19). This is a contradiction. Hence when $\varphi$ is non-zero, $p \geq q$. q.e.d.

(4.3) Let $V$ be a non-singular projective variety of dimension $n$ defined over $C$. Let $E$ be a vector bundle of rank $r \leq n$. Let $s$ be a section of $E . \quad s$ is said to be a regular section if $S=\{z \in V \mid s(z)=0\}$ is nonsingular and of codimension $r$. Griffiths [4] proved the following vanishing theorem.

THEOREM (4.3.1)*) If $E$ is positive, $r=2$ and if $E$ has a regular section, then $H^{i}(V, \breve{E})=0$ for $i \leq n-2$.

His proof depends on the generalized Lefschetz theorem and the Hodge decomposition. In fact he compared the cohomology group of $V$ and $S$ with coefficients in $Z$ by using Morse theory. But to obtain the vanishing theorem, we need only the generalized Lefschetz theorem with coefficients in $\boldsymbol{C}$. We remark here that the generalized Lefschetz theorem with coefficients in $C$ is proved by a standard technique using cohomological dimension and de Rham cohomology.

LEMMA (4.3.2) Let $V$ be a non-singular projective variety of dimension $n$ defined over $C$. Let $E$ be a vector bundle of rank $r \leq n$. Let $S$ be a zero locus of a regular section $s$. If $E$ is positive, then $V-S$ is $r$-complete. In particular ancd $(V-S) \leq r-1$ where ancd $(V-S)$ denotes the analytic cohomogical dimension of $V-S$ (cf. Umemura [16]).

*) The author learned that a more general result is obtained by J. Le Potier: Théorème d'annulation en cohomologie, C. R. Acad. Sc. Paris, t. 276 (12 février 1973) Serie A 535. 
Proof. Let $h$ be a metric on $E$. Consider the function $\varphi(z)=$ $\overline{{ }^{t} s(z)} h(z) s(z)$ on $V-S$. Since $\varphi(z)>0$ on $V-S$, we can put $\psi(z)=$ $-\log \varphi(z)$. We shall calculate the Levi form of $\psi(z)$ at a fixed point $O$ in $V \cdot-S$. We may assume that $h(O)=I, d^{\prime} h(O)=0$, hence we have $\Theta(O)=-d^{\prime} d^{\prime \prime} h(O)$. Then, by a direct calculation,

$$
\left(\frac{\partial^{2} \psi}{\partial z_{i} \partial \bar{z}_{j}}\right)_{o}=\left[\frac{1}{\varphi}{ }^{t} \bar{s}\left(\Theta_{i j}\right) s+\frac{1}{\varphi^{2}}\left[\left({ }^{t} \bar{s} \frac{\partial s}{\partial z_{i}}\right)\left(\frac{\partial^{t} \bar{s}}{\partial \bar{z}_{i}} s\right)-\left({ }^{t} \bar{s} s\right)\left(\frac{\partial^{t} \bar{s}}{\partial \bar{z}_{j}} \frac{\partial s}{\partial z_{i}}\right)\right]\right]_{o}
$$

It is sufficient to show that the Levi form $\left[\left(\partial^{2} \psi\right) /\left(\partial z_{i} \partial \bar{z}_{j}\right)\right]_{o}$ is positive definite on an $n-r+1$ dimensional subspace. Since $\sum_{1 \leq i, j \leq n}(1 / \varphi)^{t} \bar{s} \Theta_{i j} s \eta^{i} \bar{\eta}^{j}$ is positive definite, it suffices to show that the form in $\eta=\left(\eta^{1}, \cdots, \eta^{n}\right) \in C^{n}$

$$
\sum_{1 \leq i, j \leq n}\left[\left({ }^{t} \bar{s} \frac{\partial s}{\partial z_{i}}\right)\left(\frac{\partial^{t} \bar{s}}{\partial \bar{z}_{j}} s\right)-{ }^{t} \bar{s} s\left(\frac{\partial^{t} s}{\partial \bar{z}_{j}} \frac{\partial s}{\partial z_{i}}\right)\right]_{o} \eta^{i} \bar{\eta}^{j}
$$

vanishes on an $n-r+1$ dimensional subspace. Let $s={ }^{t}\left(s^{1}, s^{2}, \ldots, s^{r}\right)$. Consider the equation

$$
\left[\begin{array}{cccc}
\frac{\partial s^{1}}{\partial z_{1}} & \frac{\partial s^{1}}{\partial z^{2}} \cdots & \frac{\partial s^{1}}{\partial z_{n}} \\
\frac{\partial s^{2}}{\partial z_{1}} & & \vdots \\
\vdots & & \vdots \\
\frac{\partial s^{r}}{\partial z_{1}} & \cdots \cdots \cdots & \frac{\partial s^{r}}{\partial z_{n}}
\end{array}\right)_{o}\left(\begin{array}{c}
\eta^{1} \\
\vdots \\
\eta^{n}
\end{array}\right)=\lambda\left(\begin{array}{c}
s^{1} \\
\vdots \\
\vdots \\
\vdots \\
s^{r}
\end{array}\right]_{o}
$$

for some $\lambda \in \boldsymbol{C}$.

Then the dimension of the vector space. $W=\left\{\eta \in C^{n} \mid\left(\partial s^{i} / \partial z_{j}\right)_{o} \eta=\right.$ $\lambda \cdot s(O)$ for some $\lambda \in C\} \geq n-r+1$. We show (4.3.3) vanishes on W. In fact we have $\sum_{1 \leq i, j \leq n}\left({ }^{t} \bar{s} \cdot\left(\partial s / \partial z_{i}\right)\right)_{O}\left(\left(\partial^{t} s\right) /\left(\partial \bar{s}_{j}\right) \cdot s\right)_{O} \eta^{i} \bar{\eta}^{j}=|\lambda|^{2}\left({ }^{t} \bar{s}(O) s(O)\right)^{2}$ and $\sum_{1 \leq i, j \leq n}\left({ }^{t} \bar{s}(O) s(O)\left(\left(\partial^{t} \bar{s} /\left(\partial \bar{z}_{j}\right) /\left(\partial s / \partial z_{i}\right)\right) \eta^{i} \bar{\eta}^{j}=|\lambda|^{2}\left({ }^{t} \bar{s}(O) s(O)\right)^{2}\right.\right.$. Hence (4.3.3)= 0 on $W$.

The last assertion of the lemma follows from Andreotti and Grauert [1]. q.e.d.

THEOREM (4.3.4) Using the notation of lemma (4.3.2), the homomorphism

$H^{i}(V, C) \rightarrow H^{i}(S, C)$ is bijective for $i \leq n-r-1$ and injective for $i=n-r$.

Proof. Consider the spectral sequence of de Rham cohomology: 


$$
I_{1}^{p q}=H^{q}\left(V-S, \Omega^{p}\right) \Rightarrow H_{D R}^{*}(V-S)=H^{*}(V-S, C) .
$$

Since ancd $(V-S) \leq r-1$, we have:

$$
I_{1}^{p q}=0 \quad \text { if } q \geq r \quad \text { or } \quad p \geq n+1 .
$$

Hence $H^{i}(V-S, C)=0$ for $i \geq n+r$ and consequently $H^{i}(V, S ; C)=0$ for $j \leq n-r$. The theorem follows from the exact sequence of cohomology

$$
\rightarrow H^{j-1}(S, C) \rightarrow H^{j}(V, S ; C) \rightarrow H^{j}(V, C) \rightarrow H^{j}(S, C) \rightarrow H^{j+1}(V, S ; C) \rightarrow .
$$

Proof of Theorem (4.3.1). The following argument is due to Griffiths [4]. Let $I$ be the ideal sheaf of $S$ :

$$
0 \rightarrow I \rightarrow O_{V} \rightarrow O_{S} \rightarrow 0 \text {. }
$$

We have the commutative diagram

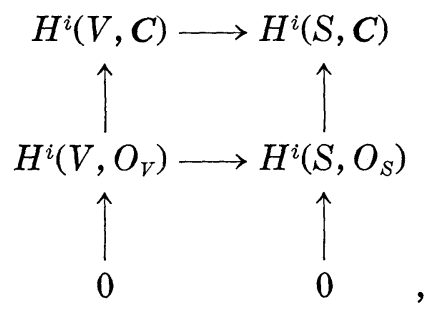

since the Hodge decomposition is functional.

By the Theorem (4.3.4), we have $H^{i}(V, I)=0$ for $i \leq n-r$. From now on we suppose that the rank $r$ of $E$ is equal to 2 . Since $s$ is regular, we can construct an exact Koszul complex by using $s$;

$$
0 \rightarrow \Lambda^{2} \check{E} \rightarrow \check{E} \rightarrow I \rightarrow 0
$$

By Kodaira vanishing theorem or Theorem (4.2), $H^{i}\left(V, \Lambda^{2} \check{E}\right)=0$ for $i \leq n-1$. Hence $H^{i}(V, \check{E})=0$ for $i \leq n-r$. q.e.d.

§. $\mathscr{G}(E)$ and $H(E)$

(5.1) Let $H$ be a finite abelian group. Let $k$ be an algebraically closed field of characteristic $p$. We consider a central extension,

$$
0 \rightarrow k^{*} \rightarrow G \rightarrow H \rightarrow 0 \text {. }
$$

Let $x, y$ be elements of $H$. We put $e(x, y)=\tilde{x}^{-1} \tilde{y}^{-1} \tilde{x} \tilde{y}$ where $\tilde{x}, \tilde{y} \in G$ lie over $x, y . \quad e(x, y)$ is an element of $k^{*}$ and is independ of the choice of $\tilde{x}$ and $\widetilde{y}$. Then $e(x, y)$ is a skew-symmetric bilinear pairing from $H$ 
to $k^{*}$. A subgroup $\tilde{K}$ of $G$ is said to be a level subgroup if $\tilde{K} \cap k^{*}=$ $\{0\}$ i.e. $\tilde{K}$ is isomorphic to its image in $H$. Let $K$ be a subgroup of $H$ such that the pairing $e(x, y)$ is trivial on $K$, then there exists a level subgroup $\tilde{K}$ lying over $K$. In fact in this case the extension is commutative over $K$ and in the category of commutative group schemes over $k$, an extension of a finite group by $k^{*}$ is trivial. If the pairing is degenerate, there exists a subgroup $K$ such that the pairing $e$ is trivial on $K$ and such that $|K|^{2}>|H|$. Hence there exists a level subgroup $\tilde{K}$ of order $>|H|^{1 / 2}$.

LEMMA (5.1.1) Let $H$ be a finite abelian group. Let $0 \rightarrow k^{*} \rightarrow G$ $\stackrel{\pi}{\longrightarrow} H \rightarrow 0$ be a central extension of $H$. If $G$ has a representation of degree 1 on which $k^{*}$ operates as the natural character, then $G$ is isomorphic to $k^{*} \times H$.

Proof. Let $V$ be a representation of $G$ of degree 1 on which $k^{*}$ operates as the natural character. We denote by $U_{x}$ the operation of $x \in G$ on $V$. Let $\tilde{K}$ be maximal level subgroup. Then there exists a character $\chi_{0} \in \operatorname{Hom}\left(\tilde{K}, k^{*}\right)$ such that $U_{x} \cdot s=\chi_{0}(x) \cdot s$ for any $x \in \tilde{K}$ and any $s \in V$. Let $y \in G$. Setting $x^{-1} y^{-1} x y=\chi^{y}(x), x \in K, \chi^{y}(x)$ is an element of $k^{*}$. $\chi^{y}: \tilde{K} \rightarrow k^{*}$ is a character. If $\pi(y) \notin \pi(\tilde{K})$, then $\chi^{y}$ is not trivial since $K$ is maximal. It is sufficient to show that $\pi(\tilde{K})=H$. Hence we have to show that $\chi^{y}$ is trivial for any $y \in G$. In fact, if $x \in \tilde{K}$, we have

$$
\begin{aligned}
\chi_{0}(x) U_{y} \cdot s & =U_{x} U_{y} s \\
& =U_{x y} \cdot s \\
& =U_{\chi^{y}(x) y x} \cdot s \\
& =\chi^{y}(x) U_{y} U_{x} \cdot s \\
& =\chi^{y}(x) U_{y} \cdot \chi_{0}(x) \cdot s \\
& =\chi^{y}(x) \chi_{0}(x) U_{y} \cdot s
\end{aligned}
$$

Hence $\chi_{0}(x)=\chi^{y}(x) \chi_{0}(x)$ and $\chi^{y}(x)=1 . \quad \chi^{y}$ is trivial. q.e.d.

(5.2) Let $A$ be an abelian variety of dimension $g$ defined over an algebraically closed field $k$ of characteristic $p$. Let $E$ be a vector bundle on $A$. We put

$$
\begin{aligned}
& H(E)=\left\{a \in A \mid E \widetilde{\rightarrow} T_{a}^{*} E \quad \text { where } \quad T_{a}: A \rightarrow A\right\} \\
& x \mapsto x+a \\
& \mathscr{G}(E)=\left\{(a, \varphi) \mid a \in H(E) \text { and } \varphi: E \widetilde{\rightarrow} T_{a}^{*} E\right\} .
\end{aligned}
$$


Let $(a, \varphi),(b, \psi) \in \mathscr{G}(E)$. Consider the composition $T_{a}^{*} \psi \circ \varphi: E \rightarrow T_{a}^{*} E$ $\stackrel{T_{b} * \psi}{\longrightarrow} T_{a}^{*} T_{b}^{*} E=T_{a+b}^{*} E$. If we define $(b, \psi) \circ(a, \varphi)=\left(a+b, T_{a}^{*} \psi \circ \varphi\right)$, then $\mathscr{G}(E)$ is a group. We have an exact suquence

$$
1 \rightarrow \operatorname{Aut} E \rightarrow \mathscr{G}(E) \rightarrow H(E) \rightarrow 0 .
$$

Let $x=(\alpha, \varphi) \in \mathscr{G}(E)$. Then $U_{x}: H^{0}(A, E) \rightarrow H^{0}(A, E)$ defined by $U_{x} s=$ $T_{-a}^{*}(\varphi(s))$ is a representation of $\mathscr{G}(E)$.

(5.2.1) If $E=L$ is of rank 1 and ample, the structure of $\mathscr{G}(L)$ is well known and $\mathscr{G}(L)$ plays a very important role in the algebraic theory of theta functions. We recall some basic properties of $\mathscr{G}(L)$ (For the details see Mumford [7]). From now on, for simplicity we assume that $\operatorname{ch} k=$ $p$ is zero. However all the results hold in positive characteristic if we avoid inseparable isogenies.

$H(L)$ is a finite subgroup of $A$. Since Aut $L \simeq k^{*}$, we have the exact sequence studied in (5.1) and (5.1.1):

$$
0 \rightarrow k^{*} \rightarrow \mathscr{G}(L) \rightarrow H(L) \rightarrow 0
$$

By the Riemann-Roch theorem, there exists an integer $d$ such that $\operatorname{dim} H^{0}\left(X, L^{\otimes n}\right)=d \cdot n^{g}$ for all $n \geq 1$. Let $\hat{A}$ be the dual of $A$. We define $\Lambda(L): A \rightarrow \hat{A}$ by sending $x$ to $T_{x}^{*} L \otimes L^{-1}$. Then we have

$$
d^{2}=|\chi(L)|^{2}=\operatorname{degree} \Lambda(L)=|H(L)| \text {. }
$$

Given a level subgroup $\tilde{K} \subset \mathscr{G}(L), L$ descends to $A / K$ i.e. there exists an invertible sheaf $L^{\prime}$ on $A / K$ such that $p^{*} L^{\prime} \simeq L$ where $p$ is the projection $p: A \rightarrow A / K$. Conversely let $K$ be a finite subgroup of $A$, the descent data associated to $L$ is given by a level subgroup lying over $K$.

Proposition (5.2.2) There exist a subgroup $K$ of $H(L)$ and an invertible sheaf $L^{\prime}$ on $A / K$ such that $\chi\left(L^{\prime}\right)=\left|H\left(L^{\prime}\right)\right|=1$ and $p^{*} L^{\prime} \simeq L$ where $p$ is the projection $A \rightarrow A / K$.

THEOREM (5.2.3) $H^{0}(A, L)$ is the unique irreducible representation of $\mathscr{G}(L)$ in which $k^{*}$ operates by its natural character.

What is $\mathscr{G}(E)$ for a vector bundle $E$ on $A$ ?

(5.2.4) Assume $A$ is of dimension 1, an elliptic curve. Let $E$ be an ample irreducible vector bundle of rank $r$ and of degree $d$, in Atiyah's 
notation, $E \in E(r, d)$. First we assume $r, d$ are coprime. Then $E$ is stable and in particular, simple: $H^{0}(A$, End $(E)) \simeq k$. Hence we get the exact-sequence $0 \rightarrow k^{*} \rightarrow \mathscr{G}(E) \rightarrow H(E) \rightarrow 0$ and a level subgroup of $\mathscr{G}(E)$ corresponds to a descent data for $E$. By Oda [12], there exists an isogeny $p: A^{\prime} \rightarrow A$ of degree $r$ and an ample line bundle $L$ of degree $d$ on $A^{\prime}$ such that $E$ is isomorphic to the direct image $p_{*} L$ and the intersection of $\operatorname{Ker} p$ and $\operatorname{Ker} \Lambda(L)$ is just 0 . Moreover, $d=\operatorname{dim} H^{0}(A, E)=$ $\operatorname{dim} H^{0}\left(A^{\prime}, L\right)$. Since the intersection of $H(L)$ and $\operatorname{Ker} p$ is 0 , a nontrivial translation by an element of $H(L)$ induces a non-zero element of $H(E)$. Hence $H(L)$ is a subgroup of $H(E)$. We have $|H(L)|^{2}=d^{2}$, hence $|H(E)| \geq d^{2}$. There exists a level subgroup of order $\geq d$. If we had $|H(E)|>d^{2}$, then there would exists a level subgroup of order $d^{\prime}>d$. Hence there would be an isogeny $\varphi: A \rightarrow A^{\prime \prime}$ of degree $d^{\prime}$ and a vector bundle $E^{\prime}$ on $A^{\prime \prime}$ such that $\varphi^{*} E^{\prime} \simeq E$. But we have $d=\chi(A, E)=d^{\prime} \chi\left(A^{\prime}, E^{\prime}\right)$. This is a contradiction. Hence $H(E)=H(L)$ and $\mathscr{G}(E)$ is nothing but $\mathscr{G}(L)$ and the unique representation of $\mathscr{G}(E)$ is given by $H^{0}(A, E)$. There exists an isogeny $\psi: A \rightarrow B$ and a vector bundle $E^{\prime \prime}$ on $B$ such that $\chi\left(E^{\prime \prime}\right)=1$ and $E$ is isomorphic to the inverse image $\psi^{*} E^{\prime \prime}$.

(5.2.4.1) In other words the theory of $\mathscr{G}(E)$ for a stable bundle over an elliptic curve is absorbed in the theory of the usual Heisenberg group and its representation.

If $r, d$ are not coprime, then $H(E)$ is too small it is not useful to consider $\mathscr{G}(E)$. Say $r=d=2$. Then by Atiyah [2], $E$ is isomorphic to $F_{2} \otimes L$ where $L$ is a line bundle of degree 1 and $F_{2}$ is the non-trivial extension uniquely determined up to isomorphism by the exact sequence $0 \rightarrow O \rightarrow F_{2} \rightarrow O \rightarrow 0$ with $\Gamma\left(A, F_{2}\right) \neq 0 . \quad F_{2}$ is homogeneous : $F_{2} \simeq T_{a}^{*} F_{2}$ for any $a \in A$. Hence if $x \in H(E)$, we have $F_{2} \otimes L \simeq E \simeq T_{x}^{*}\left(F_{2} \otimes L\right) \simeq$ $T_{x}^{*} F_{2} \otimes T_{x}^{*} L \simeq F_{2} \otimes T_{x}^{*} L$. It follows that $L \simeq T_{x}^{*} L$. In fact consider the diagram

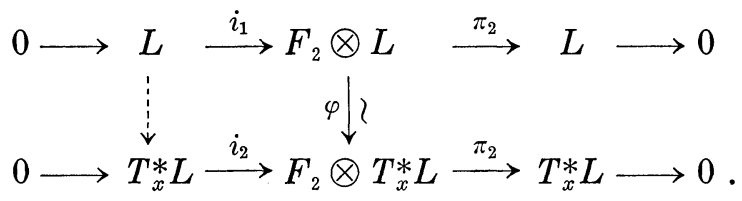

If $L$ and $T_{x}^{*} L$ were not isomorphic, then the composite $\pi_{2} \circ \varphi \circ i_{1}: L \rightarrow T_{x}^{*} L$ would be trivial since $H^{0}\left(L^{-1} \otimes T_{x}^{*} L\right)=0$. Hence $\varphi \circ i_{1}$ would factor 
through $T_{x}^{*} L$. And $\varphi \circ i_{1}$ would be injective. But this is impossible since $H^{0}\left(L^{-1} \otimes T_{x}^{*} L\right)=0$.

We proved that $H(E)=H(L)$. On the other hand by (5.2.1), we have $|H(L)|^{2}=1^{2}=1$. $H^{0}(A, E)$ is two dimensional. Hence $H(E)$ is too small.

This shows that the group $\mathscr{G}(E)$ does not give sufficient information concerning $E$ for a general vector bundle $E$. We have to restrict ourselves to a certain appropriate family of vector bundles. Over an elliptic curve, as we have seen above, the family of stable vector bundles is nice. So it is natural to ask if the group $\mathscr{G}(E)$ has good properties for $H$-stable bundles over an abelian surface. Unfortunately, the answer is no.

EXAmple (5.2.5) Let $A$ be an abelian surface. Let $E$ be an ample vector bundle of rank 2 over $\mathrm{A}$. Let $H$ be an ample line bundle on $A$. We assume that $E$ is $H$-stable.

(5.2.5.1) In the case $c_{1}^{2}-4 c_{2} \geq 0$ (This automatically is $=0$ ), since $E$ is simple, there exist an isogeny $A^{\prime} \stackrel{\pi}{\longrightarrow} A$ and an ample line bundle $L$ on $A^{\prime}$ such that $E$ is isomorphic to the direct image $\pi_{*} L$. By the same argument as in (5.2.4), we conclude that $\mathscr{G}(E)$ is nothing but the Heisenberg group $\mathscr{G}(L), H^{0}(A, E)$ is the unique representation of $\mathscr{G}(E)$ and that $E$ descends to $E^{\prime}$ with $\chi\left(E^{\prime}\right)=1 . \mathscr{G}(E)$ is considered to be reasonable.

EXAMPLE (5.2.6) We use the notation of Example (3.3). We substitute $n_{1}=n_{2}=0, m_{1}=1, m_{2}=-1$ and we consider a non-trivial extension :

$0 \rightarrow O \rightarrow E \rightarrow M_{1} \otimes M_{2}^{-1} \rightarrow 0$. Then $E$ is $H$-stable for a certain ample line bundle $H$. Let $L=M_{1} \otimes M_{2}$. Let $q$ be a prime number. Tensoring $L^{\otimes q^{n}}$ with the exact sequence above, we get an exact sequence

$$
0 \rightarrow L^{\otimes q^{n}} \rightarrow E \otimes L^{\otimes q^{n}} \rightarrow M_{1}^{\otimes\left(q^{n}+1\right)} \otimes M_{2}^{\otimes\left(q^{n}-1\right)} \rightarrow 0 .
$$

If $n \geq 2$, then $E \otimes L^{\otimes q^{n}}$ is ample and $\operatorname{dim} H^{0}\left(E \otimes L^{\otimes q^{n}}\right)=q^{2 n}+$ $\left(q^{n}+1\right)\left(q^{n}-1\right)=2 \cdot q^{2 n}-1$.

Let $x \in H\left(E \otimes L^{\otimes q^{n}}\right)$ i.e. $E \otimes L^{\otimes q^{n}} \simeq T_{x}^{*}\left(E \otimes L^{\otimes q^{n}}\right)$.

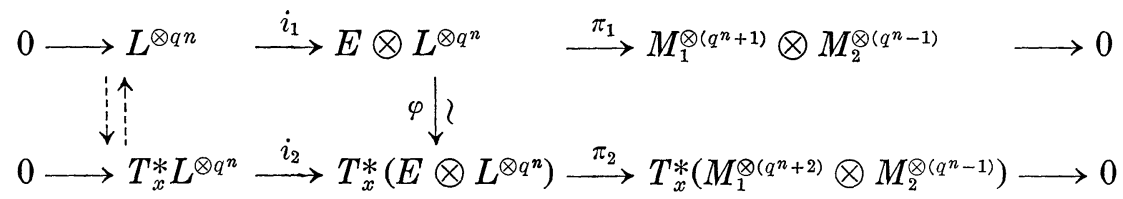

Since the index of $L^{\otimes-q^{n}} \otimes T_{x}^{*}\left(M_{1}^{\otimes\left(q^{n}+1\right)} \otimes M_{2}^{\otimes\left(q^{n}-1\right)}\right)$ is 1 , we have 
$H^{0}\left(L^{\otimes-q^{n}} \otimes T_{x}^{*}\left(M_{1}^{\otimes\left(q^{n}+1\right)} \otimes M_{2}^{\otimes\left(q^{n}-1\right)}\right)\right)=0 . \quad$ It follows that $\pi_{2} \circ \varphi \circ i_{1}=0$. Hence $\varphi \circ i_{1}$ factors through $T_{x}^{*} L^{\otimes q^{n}}$. For the same reason, $\varphi^{-1} \circ i_{2}$ factors through $T_{x}^{*} L^{\otimes q^{n}}$. Hence $\varphi$ induces isomorphisms $L^{\otimes q^{n}} \widetilde{\rightarrow} T_{x}^{*} L^{\otimes q^{n}}$ and $M_{1}^{\otimes\left(q^{n}+1\right)} \otimes M_{2}^{\otimes\left(q^{n}-1\right)}$ $\widetilde{\rightarrow} T_{x}^{*}\left(M_{1}^{\otimes\left(q^{n}+1\right)} \otimes M_{2}^{\otimes\left(q^{n}-1\right)}\right)$. This proves $x \in H\left(L^{\otimes q^{n}}\right) \cap H\left(M_{1}^{\otimes\left(q^{n}+1\right)} \otimes M_{2}^{\otimes\left(q^{n}-1\right)}\right)$ and so $H\left(E \otimes L^{\otimes q^{n}}\right) \subset H\left(L^{\otimes q^{n}}\right) \cap H\left(M_{1}^{\otimes\left(q^{n}+1\right)} \otimes M_{2}^{\otimes\left(q^{n}-1\right)}\right)$. On the other hand,

$$
\begin{aligned}
& \left|H\left(L^{\otimes q^{n}}\right)\right|=h^{0}\left(L^{\otimes q^{n}}\right)^{2}=q^{4 n} \\
& \left|H\left(M_{1}^{\otimes\left(q^{n}+1\right)} \otimes M_{2}^{\otimes\left(q^{n}-1\right)}\right)\right|=h^{0}\left(M_{1}^{\otimes\left(q^{n}+1\right)} \otimes M_{2}^{\otimes\left(q^{n}-1\right)}\right)^{2}=\left(q^{2 n}-1\right)^{2} .
\end{aligned}
$$

Hence $\mid\left(H\left(L^{\otimes q^{n}}\right) \mid\right.$ and $\left|H\left(M_{1}^{\otimes\left(q^{n}+1\right)} \otimes M_{2}^{\otimes\left(q^{n}-1\right)}\right)\right|$ are coprime and consequently $H\left(L^{\otimes q^{n}}\right) \cap H\left(M_{1}^{\otimes\left(q^{n}+1\right)} \otimes M_{2}^{\otimes\left(q^{n}-1\right.}\right)=\{0\}$.

We proved $H^{0}\left(A, E \otimes L^{\otimes q^{n}}\right)=2 \cdot q^{2 n}-1$ and $H\left(E \otimes L^{q^{n}}\right)=\{0\}$ for $n$ $\geq 2$. $\mathscr{G}(E)$ is too small in this case.

Remark (5.2.7) In the example above $c_{1}^{2}\left(E \otimes L^{\otimes q^{n}}\right)-c_{2}\left(E \otimes L^{\otimes q^{n}}\right)<0$ and the stability of $E$ depends on the choice of an ample line bundle. Again, by the conclusion that we have deduced above, the notion of an $H$-stable vector bundle with $c_{1}^{2}-4 c_{2}<0$ is not very agreeable (See Example (3.3))

Remark (5.2.8). Let $A$ be an abelian surface. Let $E$ be a vector bundle of rank 2 on $A$. The following are equivalent.

(1) $E$ is $H$-stable for any ample line bundle $H$ on $A$ and $c_{1}^{2}-4 c_{2}$ $=0$

(2) $E$ is $H$-stable for an ample line bundle $H$ on $A$ and $c_{1}^{2}-4 c_{2}$ $={ }_{6}^{0}$

(3) $E$ is simple and $c_{1}^{2}-4 c_{2}=0$

(4) There exist an isogeny $\pi: A^{\prime} \rightarrow A$ of abelian surfaces and an ample line bundle $L^{\prime}$ on $A^{\prime}$ such that $\operatorname{Ker} \Lambda\left(L^{\prime}\right) \wedge \operatorname{Ker} \pi=\{0\}$ and $E$ is isomorphic to the direct image $\pi_{*} L^{\prime}$.

(5) $E$ is simple. For any ample line bundle $L$ on $A$ and for sufficiently large $n$, we have

$$
0 \rightarrow k^{*} \rightarrow \mathscr{G}\left(E \otimes L^{n}\right) \rightarrow H\left(E \otimes L^{\otimes^{n}}\right) \rightarrow 0
$$

is a Heisenberg group: the pairing $e(x, y)$ is non-degenerate and

$$
\left|H\left(E \otimes L^{\otimes n}\right)\right|=\frac{1}{4} h^{0}\left(L^{\otimes 2 n} \otimes \operatorname{det} E\right)^{2} .
$$

(6) $E$ is simple. The same assertion as in (5) holds for one ample line bundle $L$ and for infinitely many $n>0$. 
Proof. The equivalence of (1), (2), (3) and (4) follows from Takemoto [14] and Oda [13] if we note that an extension of $O_{A}$ by $O_{A}$ is not a simple vector bundle.

We prove that (4) $\Rightarrow(5)$. Let $E$ be a simple vector bundle and let $H$ be an ample line bundle. By (4) $E \otimes L^{\otimes n}$ is isomorphic to the direct image of $L^{\prime} \otimes \pi^{*} L^{\otimes n}$. Let $x \in \operatorname{Ker} \pi \wedge \operatorname{Ker} \Lambda\left(L^{\prime} \otimes \pi^{*} L^{\otimes^{n}}\right)$. Then $L^{\prime} \otimes \pi^{*} L^{\otimes n}$ $\simeq T_{x}^{*}\left(L^{\prime} \otimes \pi^{*} L^{\otimes n}\right) \cong T_{x}^{*} L^{\prime} \otimes T_{x}^{*} \pi^{*} L^{\otimes n} \widetilde{\rightarrow} T_{x}^{*} L^{\prime} \otimes \pi^{*} L^{\otimes n}$. Hence $L^{\prime} \widetilde{\rightarrow} T_{x}^{*} L^{\prime}:$ $x \in \operatorname{Ker} \pi \wedge A\left(L^{\prime}\right)$. It follows that $x=0$. As in (5.2.4), we get

$$
\left|H\left(L^{\prime} \otimes \pi^{*} L^{\otimes n}\right)\right| \leq\left|H\left(E \otimes L^{\otimes n}\right)\right| .
$$

By descent theory (cf. (5.2.4)), we conclude that $H\left(L^{\prime} \otimes \pi^{*} L^{\otimes n}\right) \widetilde{\rightrightarrows} H\left(E \otimes L^{\otimes n}\right)$ and $\left|H\left(E \otimes L^{\otimes n}\right)\right|=h^{0}\left(E \otimes L^{\otimes n}\right)^{2}$ if $L^{\prime} \otimes L^{\otimes n}$ is ample. By the RiemannRoch theorem

$$
\begin{aligned}
h^{0}\left(E \otimes L^{\otimes n}\right) & =\chi\left(E \otimes L^{\otimes n}\right)=\frac{1}{2} c_{1}^{2}\left(E \otimes L^{\otimes n}\right)-c_{2}\left(E \otimes L^{\otimes n}\right) \\
& =\frac{1}{2} c_{1}^{2}\left(E \otimes L^{\otimes n}\right)-\frac{1}{4} c_{1}^{2}\left(E \otimes L^{\otimes n}\right)=\frac{1}{4} c_{1}^{2}\left(E \otimes L^{\otimes n}\right) \\
& =\frac{1}{4}\left(L^{\otimes 2 n} \otimes \operatorname{det} E\right)^{2}=\frac{1}{2} h^{0}\left(L^{\otimes 2 n} \otimes \operatorname{det} E\right) .
\end{aligned}
$$

Since (6) is a special case of (5), (5) $\Rightarrow(6)$ is trivial.

Now we show that $(6) \Rightarrow(3)$. Let $E$ be a simple vector bundle of rank 2. We assume that (6) holds. Then there exist an integer $n$ and an ample line bundle $H$ on $A$ such that $H^{0}\left(A, E \otimes L^{\otimes n}\right) \neq 0, H^{i}\left(A, E \otimes L^{\otimes n}\right)$ $=0$ for $i=1,2,0 \rightarrow k^{*} \rightarrow \mathscr{G}\left(E \otimes L^{\otimes n}\right) \rightarrow H\left(E \otimes L^{\otimes n}\right) \rightarrow 0$ is a Heisenberg group and $\left|H\left(E \otimes L^{\otimes n}\right)\right|=\frac{1}{4} h^{0}\left(L^{\otimes 2 n} \otimes \operatorname{det} E\right)^{2}$. Since $E \otimes L^{\otimes n}$ is simple, by the Riemann-Roch theorem, we have $c_{1}^{2}\left(E \otimes L^{\otimes n}\right)-4 c_{2}\left(E \otimes L^{\otimes n}\right) \leq 0$. Hence

$$
\text { (*) } \begin{aligned}
1 & \leq h^{0}\left(E \otimes L^{\otimes n}\right)=\frac{1}{2} c_{1}^{2}\left(E \otimes L^{\otimes n}\right)-c_{2}\left(E \otimes L^{\otimes n}\right) \leq \frac{1}{4} c_{1}^{2}\left(E \otimes L^{\otimes n}\right) \\
& =\frac{1}{2} h^{0}\left(L^{\otimes 2 n} \otimes \operatorname{det} E\right) .
\end{aligned}
$$

Since $\mathscr{G}\left(E \otimes L^{\otimes n}\right)$ is a Heisenberg group and $h^{0}\left(E \otimes L^{\otimes n}\right)$ is a representation of $\mathscr{G}\left(E \otimes L^{\otimes n}\right)$ in which $k^{*}$ operates by its natural character, $h^{0}\left(E \otimes L^{n}\right)$ is divisible by $\mid H\left(\left.E \otimes L^{\otimes n}\right|^{1 / 2}=\frac{1}{2} h^{0}\left(L^{\otimes 2 n} \otimes \operatorname{det} E\right)\right.$. By inequality $\left.{ }^{*}\right)$, we get $h^{0}\left(E \otimes L^{\otimes n}\right)=\frac{1}{2} h^{0}\left(L^{\otimes 2 n} \otimes \operatorname{det} E\right)$. Hence $\frac{1}{2} c_{1}^{2}\left(E \otimes L^{\otimes n}\right)-c_{2}\left(E \otimes L^{\otimes n}\right)=$ $\frac{1}{4} c_{1}^{2}\left(E \otimes L^{\otimes n}\right): c_{1}^{2}\left(E \otimes L^{\otimes n}\right)-4 c_{2}\left(E \otimes L^{\otimes n}\right)=0$. If follows that $c_{1}^{2}(E)-4 c_{2}(E)$ $=0$. This completes the proof.

ExAmple (5.2.9) Let $A$ be an abelian surface. Let $L$ be a very ample line bundle over $A$. Let $\varphi=\left(\varphi_{1}, \varphi_{2}, \varphi_{3}\right) \in H^{0}(A, L)^{\oplus 3}$. We assume 
that $\varphi_{1}, \varphi_{2}, \varphi_{3}$ do not vanish simultaneously. This we can define the vector bundle $E(\varphi)$, frequently referred to as Kleiman's example, by the exact sequence

$$
\begin{aligned}
0 \rightarrow O & \rightarrow L^{\oplus 3} \rightarrow E(\varphi) \rightarrow 0 . \\
1 & \mapsto\left(\varphi_{1}, \varphi_{2}, \varphi_{3}\right)
\end{aligned}
$$

LemMA (5.2.10) $H^{2}(A, E(\varphi) \otimes \check{L})=0$.

Proof. $\quad 0 \rightarrow \check{E}(\varphi) \rightarrow \check{L} \check{L}^{\oplus 3} \rightarrow O \rightarrow 0$.

$$
0 \rightarrow \check{E}(\varphi) \otimes L \rightarrow O^{\oplus 3} \rightarrow L \rightarrow 0 \text {. }
$$

Hence we get

$$
0 \rightarrow H^{0}(A, \check{E}(\varphi) \otimes L) \rightarrow H^{0}(A, O)^{\oplus 3} \rightarrow H^{0}(A, L) \rightarrow
$$

The map $H^{0}(A, O)^{\oplus 3} \rightarrow H^{0}(L)$ which sends $(1,0,0) \mapsto \varphi_{1},(0,1,0) \mapsto \varphi_{2},(0,0,1)$ $\mapsto \varphi_{3}$ is injective. Hence $H^{0}(A, \check{E}(\varphi) \otimes L)=0$. Applying Serre duality, we get the lemma.

q.e.d.

LEMMA (5.2.11) $E(\varphi)$ is simple.

Proof. Tensoring $E(\varphi)$ with the exact sequence $\left(^{* *}\right)$, we get the exact sequence,

$$
0 \rightarrow E(\varphi) \otimes \check{E}(\varphi) \rightarrow(E(\varphi) \otimes \check{L})^{\oplus 3} \rightarrow E(\varphi) \rightarrow 0 .
$$

The exact sequence of cohomology is :

$$
H^{1}(E(\varphi)) \rightarrow H^{2}\left(\text { End }(E(\varphi)) \rightarrow H^{2}(E(\varphi) \otimes \check{L})^{\oplus 3}\right.
$$

$H^{1}(E(\varphi)) \simeq H^{2}\left(O_{A}\right)$ is one dimensional and $H^{2}(E(\varphi) \otimes \check{L})=0$ by Lemma (5.2.10). Hence $\operatorname{dim} H^{2}($ End $(E(\varphi))) \leq 1$. By Serre duality, the lemma is proven.

LEMMA (5.2.12) The vector bundles $E(\varphi)$ and $E\left(\varphi^{\prime}\right)$ are isomorphic if and only if the vector space generated by $\varphi_{1}, \varphi_{2}, \varphi_{3}$ is equal to the vector space generated by $\varphi_{1}^{\prime}, \varphi_{2}^{\prime}, \varphi_{3}^{\prime}$ in $H^{0}(A, L)$.

Proof. Assume that the vector space generated by $\varphi_{1}, \varphi_{2}, \varphi_{3}=$ the vector space generated by $\varphi_{1}^{\prime}, \varphi_{2}^{\prime}, \varphi_{3}^{\prime}$. We have

$$
\left(\begin{array}{l}
\varphi_{1}^{\prime} \\
\varphi_{2}^{\prime} \\
\varphi_{3}^{\prime}
\end{array}\right)=B\left(\begin{array}{l}
\varphi_{1} \\
\varphi_{2} \\
\varphi_{3}
\end{array}\right) \text { with } B \in G L(3, k)
$$

$B$ defines an automorphism of $L^{\oplus 3}$ which we denote by $B$. We note that the following diagram is commutative: 


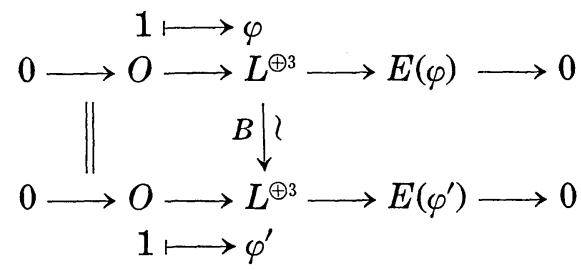

This proves the if part of the lemma.

Now we prove only if part. Suppose that $E(\varphi)$ and $E\left(\varphi^{\prime}\right)$ are isomorphic. Tensoring $L$ with the exact sequense $(* *)$, we get the diagram:

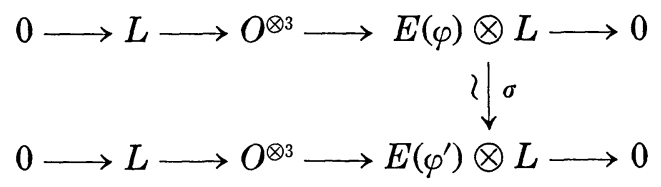

where $\sigma$ is an isomorphism. It is sufficient to show that $\sigma$ can be lifted to an automorphism of $O^{\oplus 3}$. Noting that $H^{0}(L)=H^{1}(L)=0$, we get the commutative diagram:

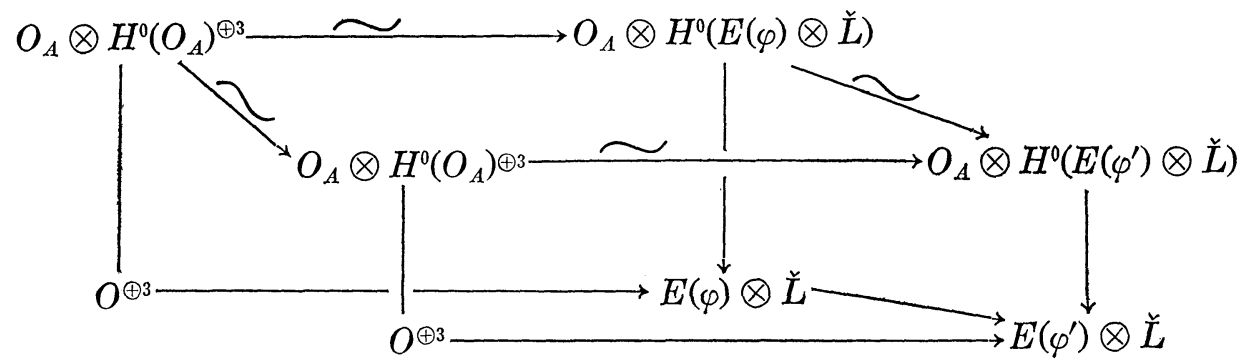

which induces an automorphism of $\mathrm{O}^{\oplus 3}$ making the diagram commutative. This completes the proof.

q.e.d.

Since $(E(\varphi))$ has a representation $H^{1}(A, E)$ of degree $1, \mathscr{G}(E(\varphi))$ is commutative by Lemma (5.1.1). We calculate $\mathscr{G}(E(\varphi))$ and its representation for a very special case. Let $C_{1}, C_{2}$ be elliptic curves. Let $A=$ $C_{1} \times C_{2}$. Let $L_{i}$ be an ample line bundle of degree divisible by 3 on $C_{i}$, $i=1,2$. Let $L$ be the tensor product $p_{1}^{*} L_{1} \otimes p_{2}^{*} L_{2}$ where $p_{i}$ denotes the projection from $C_{1} \times C_{2}$ onto the $i$-th factor. Let $\tilde{K}_{1}$ be a maximal level subgroup of $\mathscr{G}\left(L_{1}\right)$. Then there exists an element $\psi_{1} \in H^{0}\left(C_{1}, L_{1}\right)$ and an element $a$ of $\mathscr{G}\left(L_{1}\right)$ of order 3 such that $\psi_{1}$ is invariant under the operation of $K_{1}$ and $\psi_{1}, \psi_{2}=a \psi_{1}$ and $\psi_{3}=a^{2} \psi_{1}$ do not have a common zero. We do the same thing with $L_{2}$ on $C_{2}$ and we get $\psi_{1}^{\prime}, \psi_{2}^{\prime}=b \psi_{1}^{\prime}, \psi_{3}^{\prime}=b^{2} \psi_{1}^{\prime}$, $b \in \mathscr{G}\left(L_{2}\right) \quad b^{3}=1$. We put $\varphi_{1}=\psi_{1} \psi_{1}^{\prime}, \varphi_{2}=\psi_{2} \psi_{2}^{\prime}, \varphi_{3}=\psi_{3} \psi_{3}^{\prime} \varphi=\left(\varphi_{1}, \varphi_{2}, \varphi_{3}\right)$. 
Since the operations $\tilde{K}_{1} \times \tilde{K}_{2}$ and $a b, a^{2} b^{2}$ leave the vector space generated by $\varphi_{1}, \varphi_{2}, \varphi_{3}$ invariant, by Lemma (5.2.12) we have $|H(E(\varphi))| \geq 3 \times h^{0}\left(L_{1}\right)$ $\times h^{0}\left(L_{2}\right)$ where $\tilde{K}_{i}$ is the maximal level subgroup chosen above. On the other hand $\chi(E)=3 \times h^{0}\left(L_{1}\right) \times h^{0}\left(L_{2}\right)$. Since $\mathscr{G}(E)$ is commutative, $|H(E)|$ divides $\chi(E)$ by descent theory. Hence $|H(E)|=3 \times h^{0}\left(L_{1}\right) \times h^{0}\left(L_{2}\right)$. It follows that

$$
\begin{aligned}
& H(E)=Z / 3 Z \oplus \tilde{K}_{1} \oplus \tilde{K}_{2} \\
& \mathscr{G}(E)=k^{*} \oplus H(E) .
\end{aligned}
$$

Consider the exact sequence of representations of $\mathscr{G}(E(\varphi))$ :

$$
\begin{array}{lll}
0 \longrightarrow H^{0}(O) \longrightarrow H^{0}(L)^{\oplus 3} \longrightarrow H^{0}(E(\varphi)) \longrightarrow & H^{1}(O) \longrightarrow 0 \\
\text { trivial } & \text { regular } & \text { trivial } \\
\text { representation } & \begin{array}{l}
\text { representation } \\
\text { of } \boldsymbol{Z} / 3 \boldsymbol{Z} \oplus \widetilde{K}_{1} \oplus \widetilde{K}_{2}
\end{array} & \begin{array}{l}
\text { representation } \\
\text { of degree 2 }
\end{array}
\end{array}
$$

It follows that

$$
\begin{aligned}
H^{0}(E(\varphi)) \simeq & \text { regular representation of } Z / 3 Z \oplus \tilde{K}_{1} \oplus \tilde{K}_{2} \oplus \text { trivial } \\
& \text { representation of } Z / 3 Z \oplus \tilde{K}_{1} \oplus \tilde{K}_{2} \text { of degree } 1 .
\end{aligned}
$$

$E(\varphi)$ is $L$-stable.

\section{ADDED IN PROOF}

Since in Proposition (5.2) [14], the assumption that $E$ is indecomposable is forgotten, our argument in p. 111 is incomplete. We have to treat the case where $E$ is a decomposable $H$-semi-stable bundle over $A$ with $N(E) \geq 0$. In this case $E$ is isomorphic to $L_{1} \oplus L_{2}$ where $L_{i}$ is a line bundle algebraically equivalent to one another for $i=1,2$. Then it follows easily that $L_{i}$ is ample. Hence $E$ is positive in the sense of Nakano.

\section{REFERENCES}

[1] Andreotti, A. and Grauert, H. Théorème de finitude pour la cohomologie des espaces complexes, Bull. Soc. Math. France, 90 (1962), 193-259.

[2] Atiyah, M. F. Vector bundles over an elliptic curve, Proc. Lond. Math Soc. (3), 7 (1957), 414-452.

[3] Grauert, H. Über Modifikationen und exzeptionelle analytische Mengen, Math. Ann., 146 (1962), 331-368.

[ 4 ] Griffiths, P. A. Hermitian differential geometry, Chern classes, and positive vector bundles, Global Analysis, Papers in honor of K. Kodaira.

[ 5 ] Hartshorne, R. Ample vector bundles, Pull. Math. I.H.E.S., 29 (1966), 63-94.

[6] - Ample vector bundles on curves, Nagoya Math. J., Vol. 43 (1971), 73-89. 
[ 7 ] Mumford, D. On the equations defining abelian varieties I, Invent. Math., Vol. 1 (1966), 287-354.

[ 8 ] — Pathologies III, Amer. J. Math., 89 (1967), 94-104.

[9] - Abelian varieties Oxford University Press (1970).

[10] Nakano, S. On complex analytic vector bundles, J. Math. Soc. Japan, 7 (1955), 1-12.

[11] Narasimhan, M. S. and Seshachi, C. S. Stable and unitary vector bundles on a compact Riemann surface, Ann. of Math., 82 (1965), 540-567.

[12] Oda, T. Vector bundles on an elliptic curve, Nagoya Math. J., Vol. 43 (1971), 41-72.

[13] — Vector bundles on abelian surfaces, Invent. Math., Vol. 13 (1971), 247-260.

[14] Takemoto, F. Stable vector bundles on algebraic surfaces, Nagoya Math. J., Vol. 47 (1972), 29-48.

[15] - Stable vector bundles on algebraic surfaces II, in this volume, 173-195.

[16] Umemura, H. Dimension cohomologique des groupes algébriques commutatifs, Ann. scien. de l'Ecole Norm. Sup. 4 e série, t. 5 (1972), 265-276.

[17] — Cohomological dimension of group schemes, in this volume, 47-52.

[18] — Fibré vectoriels positifs sur une courbe elliptique, Bull. Soc. Math. France, 100 (1972), 431-433.

[19] Weil, A. Variétés Kähleriennes, Hermann.

[20] Hosoh, T. A paper in preparation on ample vector bundles on $\boldsymbol{P}^{2}$.

Department of Mathematics

Nagoya University 